\title{
Low-aspect ratio appendages for wind-assisted ships
}

\author{
N. J. van der Kolk ${ }^{1}$ (D) I. Akkerman ${ }^{2}$ J. A. Keuning ${ }^{2} \cdot$ R. H. M. Huijsmans ${ }^{2}$
}

Received: 15 May 2019 / Accepted: 28 September 2020 / Published online: 13 February 2021

(c) The Author(s) 2021

\begin{abstract}
Wind propulsion for commercial ships has been identified as a key component in the energy transition for the maritime industry. The sailing hybrid ship will operate with leeway (drift) angles to produce a lateral force known as sideforce, for steady operation under sail. In this paper, experimental results for the sailing performance of ships fitted with bilge keel appendages are presented. Systematic variations in appendage height, length, and position were tested, including several special cases (multiple bilge keels). The appendage typology is shown to mitigate the strong 'destabilizing' yaw moment that is characteristic of wind-assisted commercial vessels and to promote the non-linear sideforce component. The working principal for bilge keels - promotion of flow separation — can be employed to specify the separation location for components of the vessel vortex wake to improve the sailing performance of the ship.
\end{abstract}

Keywords Green ships $\cdot$ Hydro-mechanics $\cdot$ Naval architecture $\cdot$ Ship maneuvering $\cdot$ EEXI/EEDI

\section{Introduction}

Wind assist, an environmentally friendly auxiliary propulsion system for commercial ships, is identified by [31, 41-43, 46], among others, as a key intervention for the energy transition in the maritime shipping space. Windassisted ship propulsion promises substantial reduction in greenhouse gas emission in the near term. The technical and commercial viability of sailing for modern commercial vessels has been the subject of study since the OPEC oil crisis of 1973 (see, for example, [39]). At that time, progress towards real-world implementation in Japan was most advanced, culminating in a full-scale demonstration ship and 17 installed systems in the Japanese short-sea shipping fleet [49]. More recently, the researchers Fujiwara and Ueno have published a series of studies associated with a new (planned) demonstration vessel, the Wind Challenger [36], including testing of a $1 / 3$ scale prototype of a collapsible solid wing sail [14], and a presentation of towing tank results for systematic variation in appendage configurations [32]. The

N. J. van der Kolk

nvanderkolk@bluewaspmarine.com

1 Blue WASP, Strevelsweg 700-303/D5025, 3083AS Rotterdam, The Netherlands

2 Delft University of Technology, Mekelweg 2, 2628CD Delft, The Netherlands present study, concerned with the sailing performance of bilge keels, is an extension of the experimental work of [32].

\subsection{Course stability for sailing ships}

The ship hull creates hydrodynamic sideforce in reaction to the transverse component of the force created by the wind propulsor by adopting a leeway angle: an angle of attack for the hull. The sailing ship in operation with a steady leeway angle will also produce a strong hydrodynamic Munk moment about the yaw axis [33]. This destabilizing phenomenon arises when a slender body encounters an oblique flow, where the pressure distribution will tend to reorient the body perpendicular to the flow.

The center of lateral resistance (CLR) expresses the strength of the yawing moment in relation to the sideforce produced, as the centroid of the lateral load. It is measured with respect to the midship:

$\operatorname{CLR}=\frac{N}{Y}$.

The importance of vessel yaw balance, with CLR and $\partial \mathrm{CLR} / \partial \beta$ as determinants for vessel course-keeping and directional stability, and key design constraints for sailing vessel hydrodynamics, has been discussed by $[8,26,35]$ in their investigations of sailing yachts. 


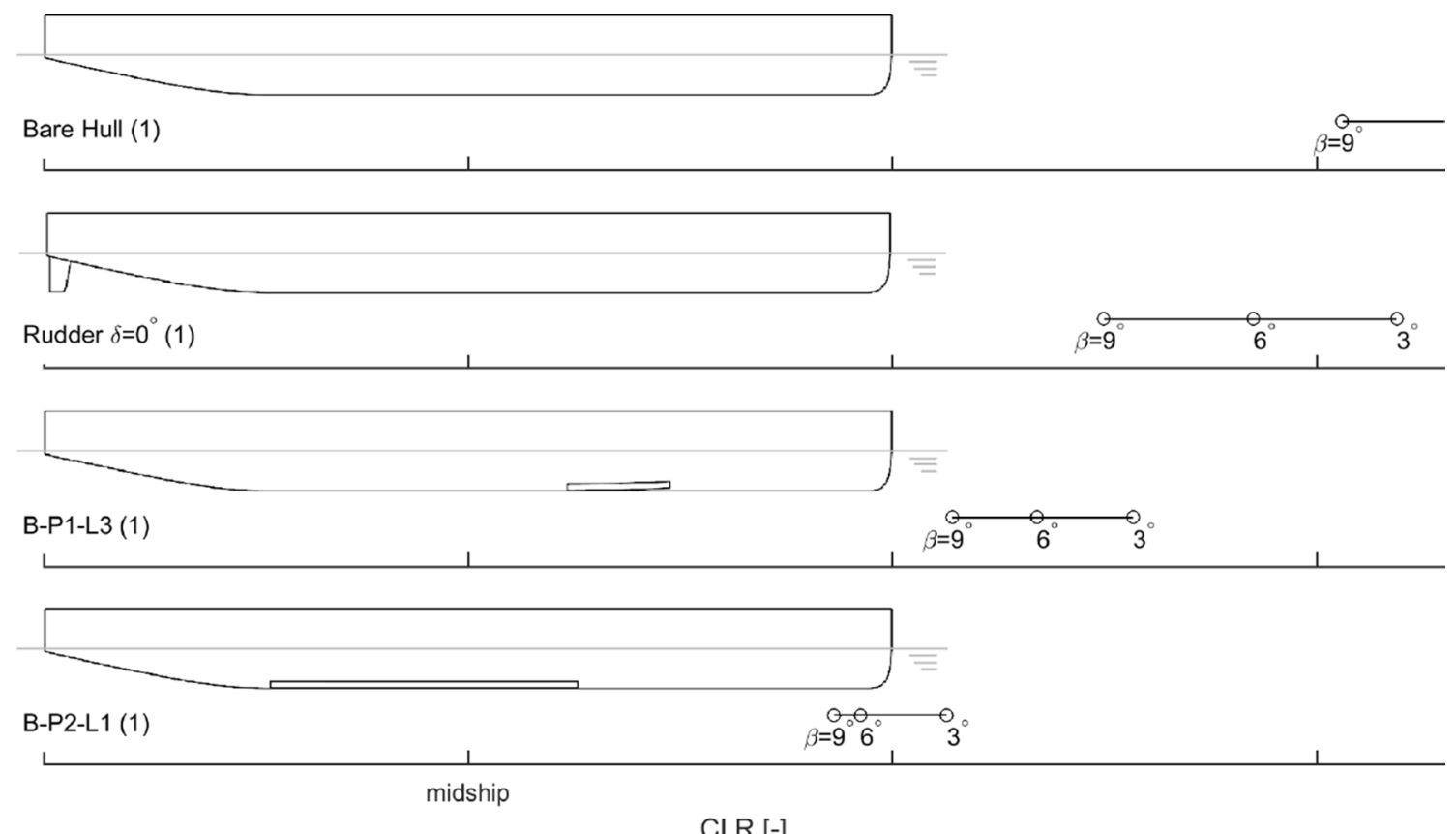

Fig. 1 Vessel CLR for several appended hulls. Experimental result

Behavior of the CLR is shown in Fig. 1 for several appendage configurations: the bare hull (un-appended), the nominal appended hull design with rudder set for $0^{\circ}$, a short bilge keel in the most forward position, and a long bilge keel occupying the full length of the parallel midbody. The CLR is given for leeway angles $\beta$ of 3,6 , and $9^{\circ}$.

First, observe the CLR for the un-appended hull, which lies more than half a ship length ahead of the bow (shown only for $9^{\circ}$ leeway), a consequence of the stronger development of the yawing moment-linear with leeway angle-compared against the sideforce, which includes a significant higher order dependency for leeway angle. The CLR moves aft as the leeway angle increases, an effect that is driven by an increase in flow separation along the bilges. This effect is manifest as a rising contribution for the higher order sideforce term in the sway equation, and an attenuation of the Munk moment for the yaw equation by flow separation along the vessel aft-body. Yaw balance, achieved by aligning the aerodynamic center of the wind propulsors with the hydrodynamic center (CLR) of the hull [8], is impossible.

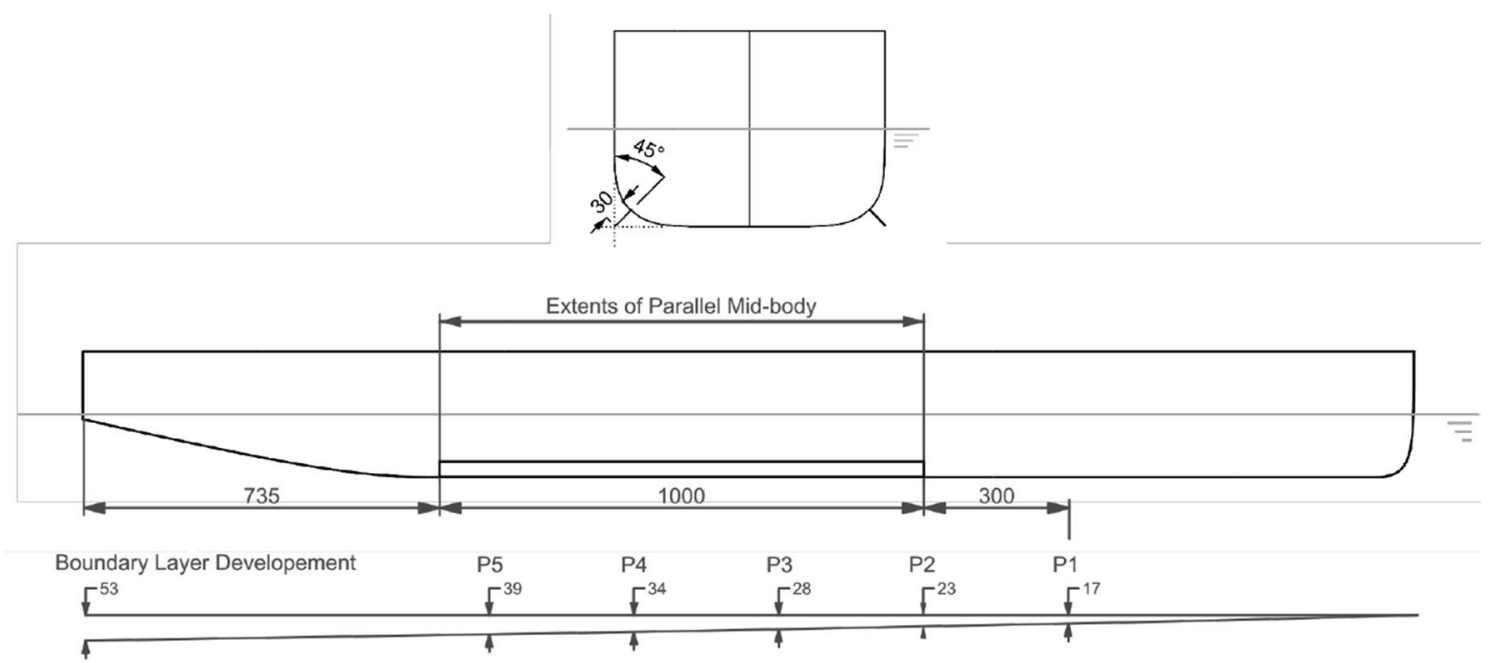

Fig. 2 Bilge keel geometry B-P2-L3-H3 (1). The estimated model-scale boundary layer development is given in mm 
For the rudder case, for $0^{\circ}$ rudder angle, the CLR remains well ahead of the ship bow, a result also reported by [13, 40]. Any corrective action by the rudder will introduce a further resistance, the induced resistance of the rudder. The slender body modeling may be applied to the ships rudder. Under the modeling usually applied when deriving mathematical models for maneuvering simulations, interaction effects, such as end-plate, and free surface effects (which might become relevant for larger heel angles) are modeled with empirical coefficients.

The rudder for a conventional cargo vessel is dimensioned to work in the wash of the propeller, where it experiences a flow that is accelerated by the working of the propeller. When wind propulsors are able to contribute thrust and the main propulsor thrust is thereby reduced, the flow velocity over the rudder is correspondingly reduced. These two effects will compound: the destabilizing moment responsible for weather helm generally increases with aerodynamic thrust, and while the rudder can be used to oppose this destabilizing moment, it will become less effective as the main propeller thrust is reduced.

The third and fourth cases shown in Fig. 1 are bilge keel geometries from the set of appendages variations tested in the present research. Both variations exhibit superior performance compared to the rudder case: the CLR is shifted aft to the close proximity of the bow. Also, the variation of CLR with leeway angle is reduced (the grouping of CLR values becomes more compact). A minimal value for the variation for the CLR with leeway angle is written as minimization for the variation in CLR for leeway $\beta$, and heel $\phi$ :

$\min \left(\nabla_{\beta \phi} \mathrm{CLR}\right)$.

The rudder angle $\delta_{\text {Rud }}$ may also be considered, as under the study of maneuvering of ships. If the linear maneuvering models that follow from slender body theory are substituted, the CLR will be constant for all leeway angles, at $L / 2$ ahead of the ship bow. The variation for the CLR with leeway angle, $\partial \mathrm{CLR} / \partial \beta$, is detrimental for vessel course keeping, where a minimal value is desired (a compact grouping for the CLR as in Fig. 1. These attributes characterize a balanced and course-stable sailing ship.

\subsection{Theoretical background}

The sailing performance for wind-assisted ships is synonymous with maneuvering forces for the steady drift condition, i.e. increase in resistance, lateral force (sideforce) production, and yaw moment due to drift (leeway, $\beta$ ) angle. Whereas the analysis of experimental results will rely also on common decompositions from the study of ship maneuvering, the nomenclature of the study of sailing is adopted in the remainder of this paper.
In general, for previous work primarily concerned with the maneuvering of ships, the drift angles considered are larger than what is considered an operational leeway angle for wind-assisted vessels. One notable exception is the experimental and theoretical studies by [2-4], where a ship-like foil with low aspect ratio was towed at angles of attack including $4^{\circ}$ and $8^{\circ}$. Wing geometries with square and rounded tips were tested, where the square tips were found to produce more lift and drag, which is understood as a result of a contribution by the crossflow component arising due to separation effects that will be more pronounced for the sharp wing tip geometry. Similar results are reported in [29]. Finally, lift and moment coefficients calculated by the added mass impulse of [24] were found to agree well with the experimental results for rounded wing-tips, but less so for the square tip profile. This finding implies that the hydrodynamic reaction is nearly linear for small angles, with a small viscous contribution arising from flow separation Beukelman and Journée [5]. Similar results are reported in $[23,27,44]$. In common engineering practice, maneuvering forces are linearized for small angles.

The bilge keel is a common appendage type that is normally applied to regulate roll motion, in particular for slowmoving ships. The cross-sectional geometry for typical commercial ships results in a roll degree-of-freedom that is particularly susceptible to underdamped resonant behavior. The promotion of flow separation is the working principle of the bilge keel, whereby the viscous damping term in the roll equation is increased. The addition of bilge keels serves to excite the eddy-making process, which is only present for bare hulls at large roll angles. This viscous damping contribution has been the subject of many investigations, beginning with that of Froude [12]. Important contributions in more recent times include the work of [22], who demonstrated that the eddy-making damping is nearly independent of Froude number, and [6,11], who applied a vortex tracking method to the problem. For the conventional application, the bilge keel is subject to an oscillating flow induced by the roll motion of the ship. This unsteady process is normally investigated in U-shaped water tunnels, as in [11,38], among others, or with an oscillating cylinder as in [45].

The present study for bilge keel and centerline keels in steady (oblique) flow corresponds to a special case of the unsteady process considered by previous researchers working on the roll damping problem. The impact of bilge keels on the manoeuvring characteristics was investigated by Yasukawa [48], where an increase linear manoeuvring derivatives was observed. The range of drift angles for a manoeuvring study $\left(5^{\circ}-20^{\circ}\right.$ in this case) is large compared to the expected range for wind-assist. Faltinsen reports results for the influence of bilge radius and bilge keel height that are reproduced in the present work. Faltinsen also addresses the scaling challenge inherent in any experimental investigation 
of bilge keels, where the working mechanism is Reynolds number dependent. This issue is addressed further under experimental design in Sect. 2.

The hydrodynamics of sailing commercial ship types is characterized by a system of shed vortices that separate from and pass around the hull, forming the wake of the ship. Vessel sailing behavior depends on the strength and separation location for these primary vortices and any secondary/counter rotating components. Many experimental and numerical investigations have explored the changes to the vortex system for varying angles of oblique flow, where again the flow angles considered are generally larger than would be considered operational leeway angles for a wind-assisted ship. From the oil film experiments of [6], to detailed measurements of the vortex wake by [30], to state-of-the-art numerical work such as the detached-eddy Reynolds-averaged Navier-Stokes simulations by Carrica et al. [7], Abdel-Maksoud et al. [1], and Xing, Bhushan, and Stern [47], the flow patterns have been well documented. As described by [30], the topology for the wake of a ship sailing with a leeway angle is characterized by several vortices, of which the forebody keel vortex (FKV), forebody bilge vortex (FBV), and forebody side vortex (FSV) are relevant here. As discussed above, the strength and separation location of the components of the vortex wake will vary with the leeway angle. For sailing ships, placement of bilge keels or centerline keels raises the possibility of specifying the separation location for components of the vortex wake, thereby manipulating the topology of the vortex system. Desirable effects, when attempting to influence the vortex wake to improve the sailing performance of the ship, are the promotion of the higher order sideforce component and the attenuation of the Munk yawing moment.

Finally, sideforce production by the sailing ship, including possible corrective action with the rudder, will introduce an added resistance to the ship arising due to a dissipation of energy in the vortex wake of the ship. Following theories for low-aspect planforms [17, 24], this induced drag may be significant for commercial ships, meaning that the thrust delivered by a wind propulsor might well be overwhelmed by this increase in resistance. Though the flow mechanisms only vaguely resemble the Prandtl lifting-line and the associated derivation for the induced drag [37], accounting for energy loss in shed vorticity is especially relevant to the present application.

Following the analysis of sailing yachts by [15], the resistance increase due to sideforce production is modelled as an effective draft, $T_{\mathrm{e}}[16]$, which is a metric for the sailing efficiency of the hull. The quantity $T_{\mathrm{e}}$ is related to the effective aspect ratio, representing the span of a wing profile with equivalent behavior for the induced drag. The modelling for effective draft has pragmatic appeal, but it must be noted that significant liberties are taken when treating a ship hull as a
Prandtl wing. For ship geometries, flow characterized as 'tip effects' in Prandtl's theory, where streamlines curl around the end of the foil in response to the pressure gradient, will influence the flow pattern over the entire span (or depth of the hull). Furthermore, a commercial hull form does not present a well-defined trailing edge, which introduces ambiguity for the definition of a circulation as it is not clear where (or whether) a Kutta condition should be applied. Nevertheless, the flow mechanisms responsible for the development of sideforce-the separation of several well-defined vortices into the wake of the ship-are dissipative processes that introduce a further resistance. This resistance penalty associated with sideforce production is an indication of the sailing efficiency of the ship, and is reported as the effective draft.

The hull is considered to be a low-aspect ratio wing with a linear sideforce written using the well-known model presented by Jones [24]. The cross-flow drag, or lateral edge vortex flow as described by Hoerner [18], is sideforce production by momentum transfer as flow separates from the hull. Hooft and Quadvlieg have related the crossflow drag component of the sideforce generated by a ship to the strength and separation location for shed vortices originating primarily along the bilges of the ship [19, 20]. Further, they have shown that the separation location will shift along the bilges for varying leeway angle, being concentrated near the bow for small leeway angles. A low-aspect rectangular planform, such as a bilge keel or a simplified hull form, develops a concentrated lifting force at the leading edge [34]. Low-aspect lift is characterized by a "sectional lift angle", whereby locations along the chord are influenced by the downwash from upstream sections of the lifting profile, and the final downwash angle is reached well before the trailing edge. For the very low aspect ratios considered in this study, for both the ship hull and the bilge keels, the linear lift coefficient is expected to be independent of the chord, corresponding to the ship length or bilge keel length.

\subsection{Present experiments}

This publication presents experimental results for the sailing performance of low-aspect ratio appendages: bilge keels and centerline keels. The experimental data are made publicly available to support further academic work and the eventual commercial uptake of wind-assist technology for commercial shipping. A discussion of main effects observed is included in the present publication. A detailed description of the model and appendage construction can be found in Sect. 2, along with a description and motivation of the experimental design. The model and appendage geometry is included at the end of this manuscript. Experimental results are presented and discussed in Sect. 3. Due to the large volume of data collected, the discussion is restricted to key 
effects. All quantities derived in this analysis are included in the data set, available in an online archive [28].

\section{Methodology}

\subsection{Experimental design}

The objective for the experimental campaign is to collect data to facilitate a modelling for the influence of bilge keels on the sailing performance of a wind-assisted ship. Bilge keel height and position are identified as the primary independent variables for the experimental design. Following the discussion of low-aspect lifting surfaces provided in Sect. 1.2, it is expected that the position of the bilge keel leading edge will dominate the hydrodynamic response. The appendage variants are arranged into sub-sets with constant aspect ratio to test to what extent this class of appendages acts as a conventional lifting surface. The behavior for such an appendage, well described by circulatory lift models, is expected to correlate with the appendage aspect ratio, defined as $\mathrm{AR}=2 h / l$. The appendage variations were located along the parallel midbody insofar as possible. Bilge keel position with respect to bare-hull separation phenomena is also of interest. The appendage positions P1, located at the forward shoulder for Hull \#1, and P5, located at the aft shoulder, are expected to interact directly with bare-hull separation phenomena.

The instigation of flow separation in the boundary layer is the working principle, and a principal challenge in experimental design is the efficacy of the bilge keel appendage in a model-scale boundary layer. The bilge-keel behavior within the rather-too-thick model scale boundary layer, and the subsequent interaction between the vortex system and the boundary layer, pose elemental difficulties in the interpretation of these results for the full-scale application. A simple geometric scaling for the bilge keel would not be appropriate, as the resulting model-scale bilge keel height would remain quite close to the inner region of the boundary layer, extending less than one third through the total boundary layer thickness (determined using $\delta_{99}$, according to the $1 / 5$ power law for a turbulent boundary layer thickness). The nominal bilge keel height used in the experiment is chosen such that the bilge keel extends unambiguously through the entire thickness of the boundary layer. A set of bilge keels with systematically varied bilge keel height was tested to control for this scaling effect, where it is understood that the flow mechanism of interest is Reynolds number dependent.

The bilge keel and centerline keel variations are defined to test for the behavior for varying centerline keel appendage length and appendage position, and to study the difference with performance with identical bilge keels and centerline keels. Appendage variations are tested on three hull models: Hull \#1 (parent hull for the Delft Wind-Assist Series) Hull \#16, which is derived from Hull \#1 by increasing the prismatic and midship coefficients, and Hull \#34, which is derived from the Ecoliner parent (hull \#1) by adding $10^{\circ}$ deadrise angle while keeping the same prismatic coefficient and draft. Hull \#16 has an elongated parallel midbody (1300 mm compared with $1000 \mathrm{~mm}$ for Hull \#1) and a reduced bilge radius (by 50\%). The centreline keel variants are tested on Hull \#34, for which the $\frac{R_{\mathrm{b}}}{T}$ is somewhat increased compared with Hull \#1.

\subsection{Experimental setup}

The following nomenclature is adopted for the appendage variants: each bilge keel variant is labeled with four numbers, specifying the longitudinal position, keel length and keel height, and finally, the hull number. For example, the bilge keel case in Fig. 2 is B-P2-L3-H3 (1) (Bilge keel, Position 2, Length 3, Height 3, Hull \#1). The bilge keel occupies the full extent of the parallel mid-body. Special cases are designated with a single number as BS1, BS2. All appendage variations and main particulars for the hulls are provided at the end of this manuscript.

The experimental campaign was conducted in the large towing tank at the Delft Ship Hydro-mechanics Laboratory at Delft University of Technology. The experimental setup was designed to facilitate quick and precise arrangement for each appendage variation. This choice was motivated by the small magnitude for the quantities of interest, a high sensitivity to model alignment and geometric errors, and an experimental design that called for multiple variations for the appendage geometry.

The hexapod and six-degree-of-freedom measurement frame are used in the same configuration as during the validation experimental campaign [28], and grids are tested for fixed trim and sinkage. Each hull model was fitted with an alignment frame designed to enable repeatable, precise mounting of the model to the six-degree-of-freedom measurement frame. The position of the model is recorded using a Certus ${ }^{\mathrm{TM}}$ optical tracking system. All signals are filtered with a low-pass filter set to $100 \mathrm{~Hz}$ before sampling to prevent aliasing. The signal is sampled at a frequency of 1000 $\mathrm{Hz}$ and written to disc. An average is made over a 40-second measurement, which is made after the carriage acceleration is complete and $10 \mathrm{~s}$ have passed to allow for the flow to reach a steady condition. The nominal rest period between runs was $20 \mathrm{~min}$. All bilge keel variants were towed at a Fn number of 0.21 . The nominal testing matrix for each bilge keel variant was defined as a combination of an equal number of positive and negative leeway angles, and the test program was arranged so that positive and negative leeway angles and any repeat runs were interspersed regularly. The 
Fig. 3 Bilge keel B-P2-L1-H3 on Hull \#1 (left) and Hull \#16 (center), and C-P2-L1-H3 on Hull \#34 (right)
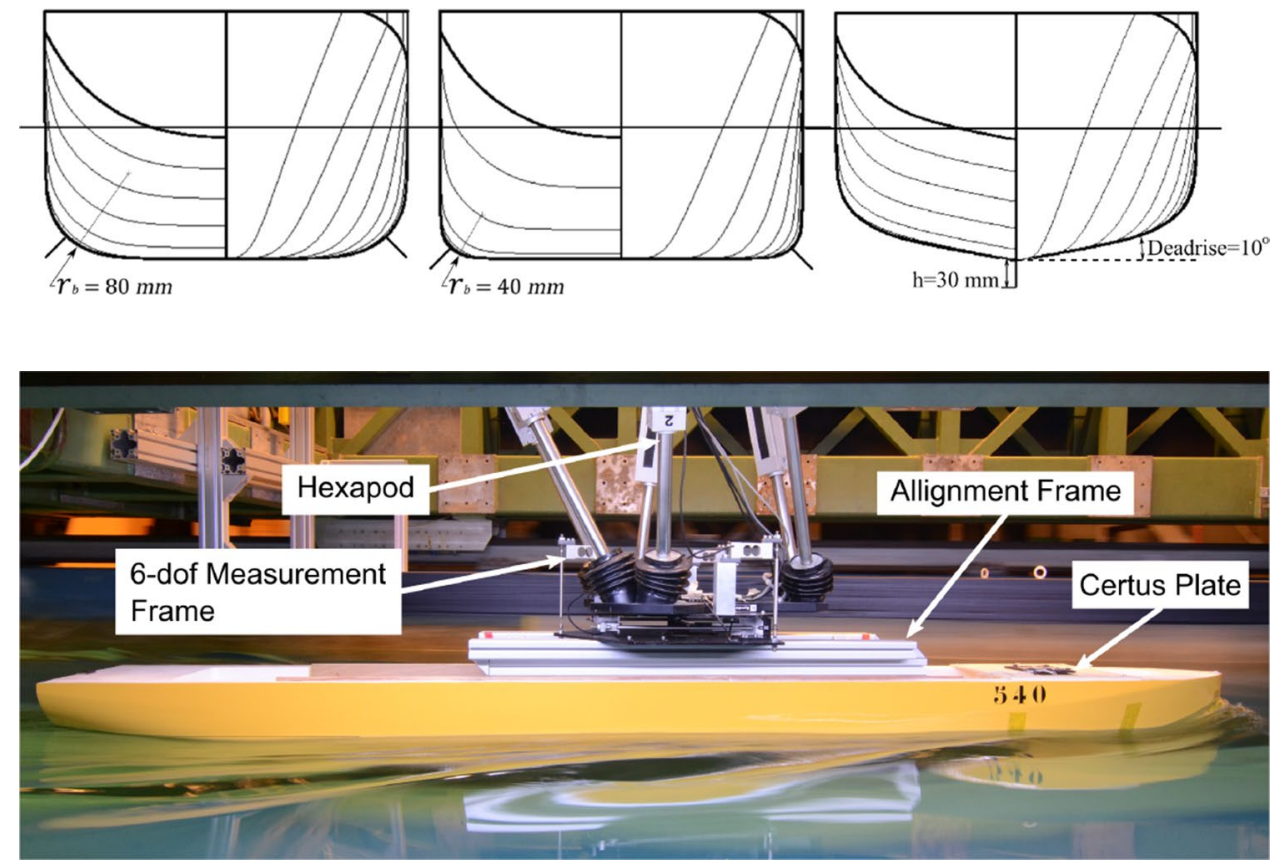

Fig. 4 Overview of experimental setup with key components labeled first run of each day, and the first run after a weekend, was marked in the measurement log.

The bilge keel geometry and bilge keel position for each variant were realized with care. The assembly of appendage B-P3-L1-H3 (16) is shown in Fig. 5. The reproducibility of the appendage geometries was tested by rebuilding and retesting bilge keel B-P3-L1-H3 (16) at the end of the experimental campaign. A set of repeat measurements was made for both realizations, as shown in Fig. 6 for the resistance. Though a distinction between the realizations of the geometry is evident, the grouping of the results is quite close. The $95 \%$ confidence interval for the complete set of 20 repeat measurements is $2.5 \%$.

\subsection{Uncertainty assessment}

The analysis for measured quantities follows a straightforward application of the towing tank standard procedure [21]. For derived quantities such as effective draft, $T_{\mathrm{e}} / T$, a more deliberate assessment of the experimental uncertainty is required. The objective in this experiment was to reveal the sailing characteristics of bilge keels, rather than to generate data suitable for validation. The uncertainty assessment is presented to attest that the measurement fidelity was sufficient for the small quantities of interest, and that the measurement setup could accommodate high sensitivity to model alignment and geometric errors whilst carrying out an experimental campaign during which the model was repeatedly removed to rebuild the bilge keel geometry. The discussion of the uncertainty for measured quantities is devoted to resistance and the induced resistance in particular, which has

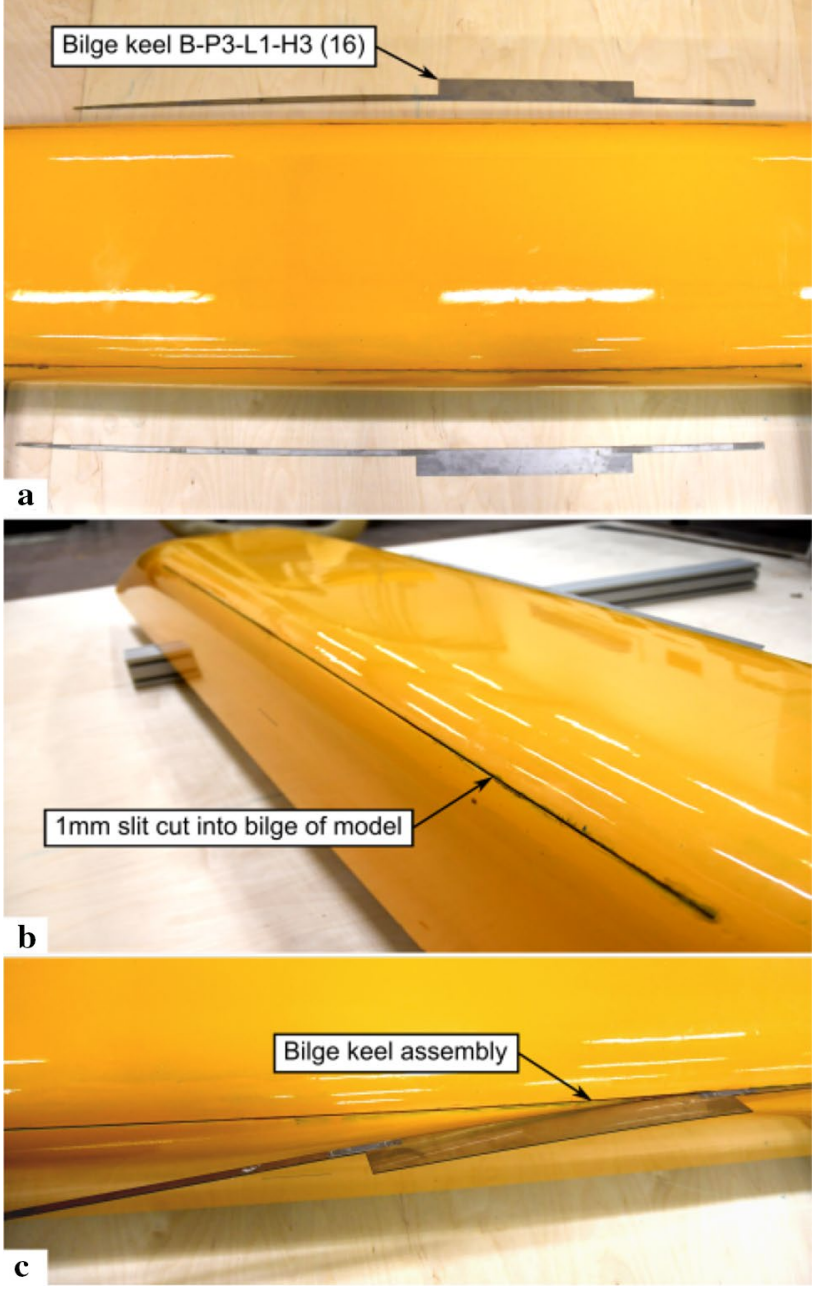

Fig. 5 Assembly of bilge keel B-P3-L1-H3 (16) 

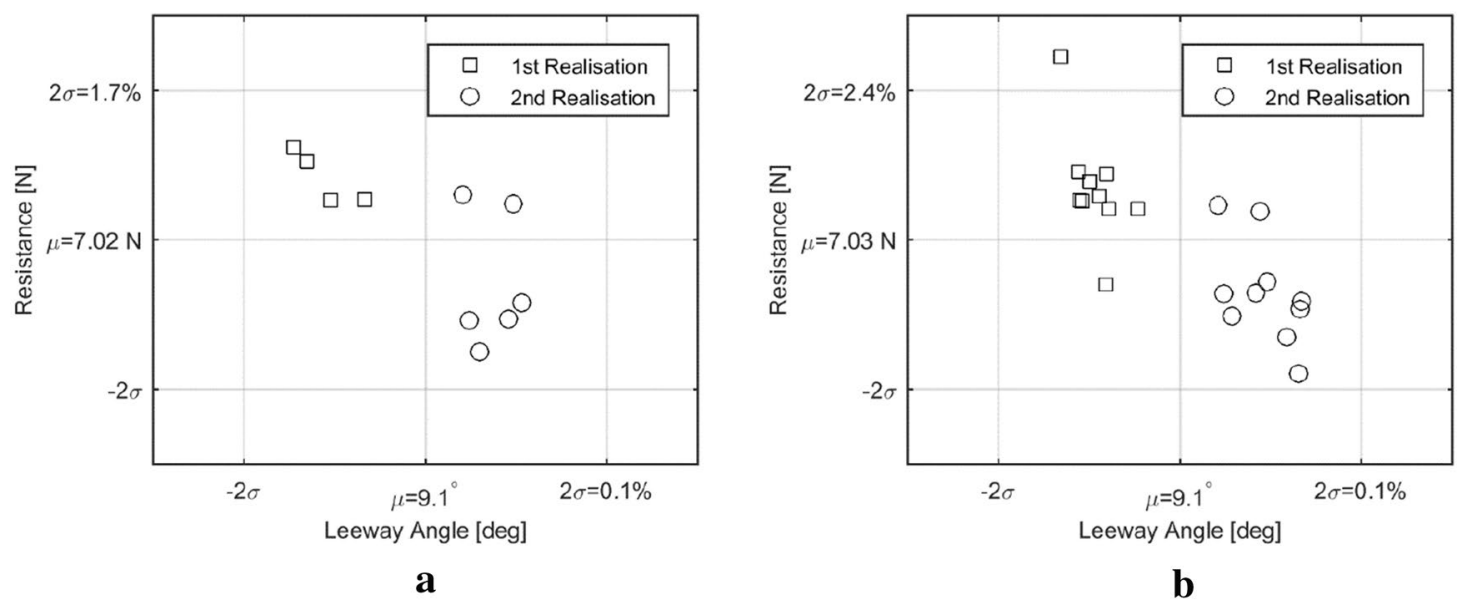

Fig. 6 a Precision limit of the alignment frame for resistance. b Complete set of repeat runs for precision limit for resistance $n=20$

been identified as the most demanding for the fidelity of the measurement system.

The following bias errors are found to be significant: calibration for the measurement frame, alignment of the model, and construction of the model geometry. Three components of the experimental system will be discussed: the design and calibration of the six degree-of-freedom measurement frame, the alignment frame, and the reproducibility of appendage geometries. A complete reporting of the uncertainty assessment is included in the data archive [28].

\subsubsection{Measurement frame}

The design of the six degree-of-freedom (6-dof) measurement frame is informed foremost by the relatively small induced resistance, compared to the other measured quantities and especially compared to the displacement of the ship model. See Fig. 4 for experimental setup. Whereas the weight of displaced water is approximately $90 \mathrm{~kg}$, the induced resistance is typically less than one Newton. The strain gauges had to accommodate the rather large inertial loading while accelerating the model, yet be able to measure variations in the induced resistance. Zemic L6J load cells with $3 \mathrm{~kg}$ capacity were used for forces measured in the lateral plane. The sensors are fitted to a 6-dof measurement frame that uses an arrangement of necked pins to decompose a loading state into orthogonal forces and moments. This arrangement of gauges and pins will introduce a further stiffness to the calibration factor of the individual gauges. Also, the mechanical complexity of the frame compounds the hysteresis effect of individual gauges. A calibration is performed after frame assembly to verify alignment of the components and to control for the effect of frame stiffness and hysteresis. The bias error, determined from the frame calibration by the SEE method [9], is reported in Table 2 for the resistance.

\subsubsection{Alignment frame}

An alignment frame was designed to enable repeated, precise mounting of the model beneath the measurement frame. The level of precision attained was measured with a series of 10 repeat measurements, for which the model was disconnected from the measurement frame before each run. The result for this test for the resistance is plotted in Fig. 6a. This result is taken as the precision limit for the experimental setup.

The model was disconnected repeatedly from the measurement system to allow construction of each bilge keel variant. Each reassembly of the measurement setup raises the possibility of model misalignment in the tank. The precision achieved with the alignment frame was sufficient to allow for a single alignment for each hull, accomplished with a set of positive and negative repeat measurements. The nominal test matrix for each appendage variant included an equal number of positive and negative leeway angels. The fitting for maneuvering coefficients and effective draft is, therefore, insensitive to (small) misalignment for the model, which would be manifest as a constant $y$-intercept in the regression fits. The bias for alignment was determined as the root-sumsquared combination of leeway angles for zero sideforce and moment — a conservative estimate.

\subsubsection{Appendage geometry}

The reproducibility of the appendage variations was tested by rebuilding and retesting case B-L3-P3-H3 (16) at the end of the campaign. The result for the resistance is plotted in Fig. 6a. The mean value and the two-sigma value are 
indicated. Results for the $95 \%$ confidence interval, based on the complete set of 20 repeat measurements, are provided in Fig. 6 and Table 1. Whereas the close grouping for these measurements lends assurance to the reproducibility of the model geometry, it would be inappropriate to derive a geometric bias contribution from this test because the difference in the measurements for the first and second realization of the bilge keel geometry may be the result of other error sources besides a geometric fault. Therefore, a geometric error is assumed based on the tolerance achieved during the preparation of the slits in the bilges of the hull models and the experience of the bilge keel assembly.

ID: B-P4-L2-H3 (16)

$$
\begin{aligned}
& C_{x_{0}}=0.025+/-0.001 \\
& x_{\beta \beta}=0.41+/-0.05
\end{aligned}
$$
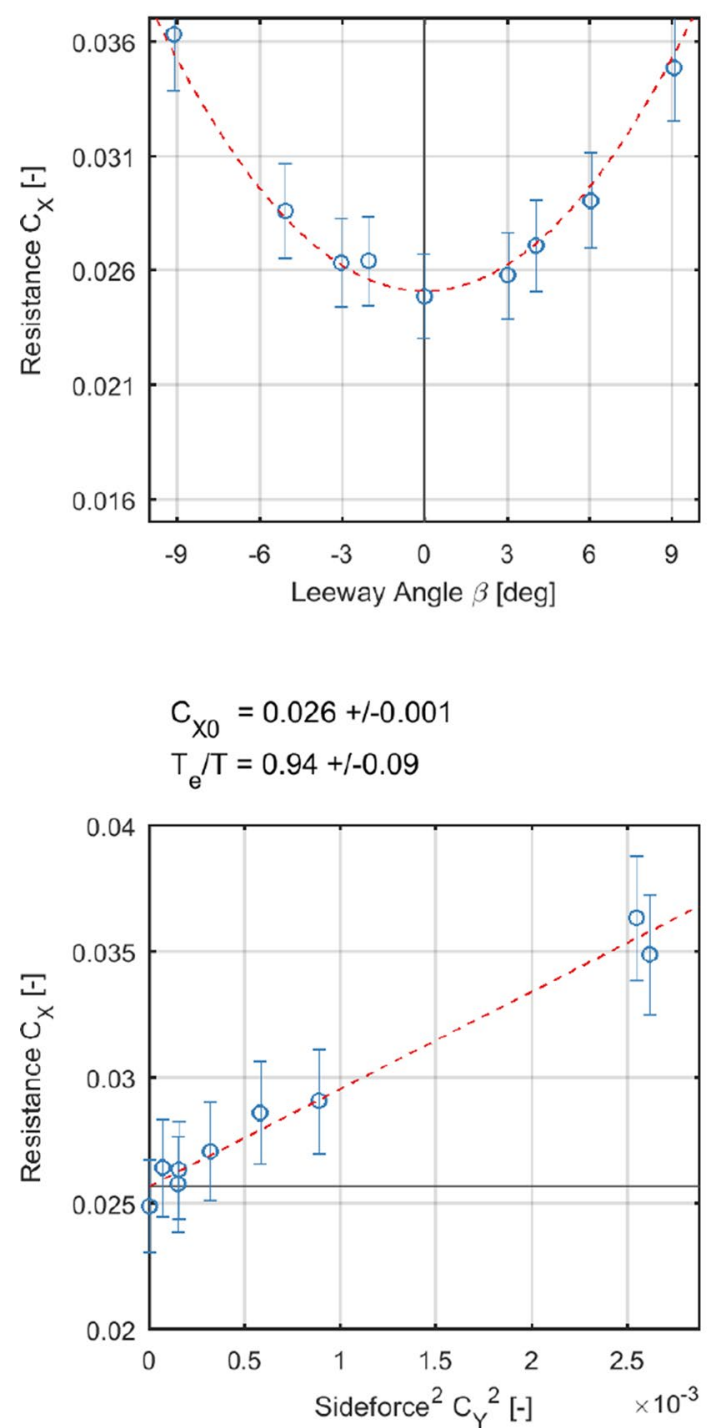

\subsubsection{Derived quantities}

The $95 \%$ confidence intervals for maneuvering coefficients, the CLR, and the effective draft are included in all figures in the discussion below. The uncertainty associated with some derived quantities is rather large. For example, the number of available data points for fitting a third-order polynomial is limiting for the higher order terms in the maneuvering equations. For the CLR, for which the uncertainty follows a direct application of uncertainty propagation rules, the relatively small sideforce in the denominator in the equation for CLR results in large sensitivity coefficient in the uncertainty
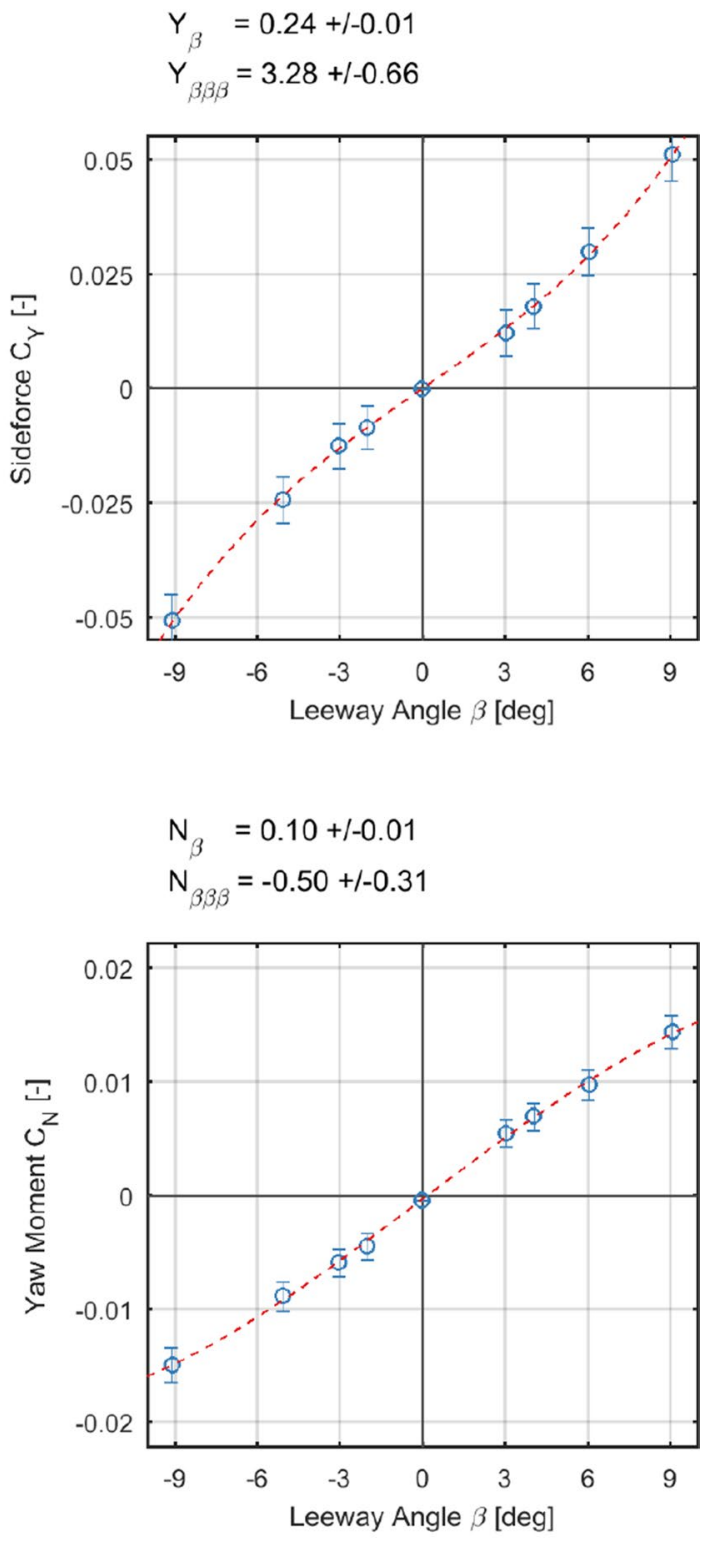

Fig. 7 Example of data reduction for bilge keel case B-L4-P2-H3 (16) showing derived quantities and associated uncertainties 
propagation. The symmetry of the testing matrix, with a distribution of positive and negative leeway angles, means the bias contribution for model orientation may be disregarded for the maneuvering coefficients and the effective draft. In light of the relative magnitude of the uncertainties of the remaining bias contributions outlined above, the uncertainty reported for maneuvering coefficients and the effective draft is simply the uncertainty associated with fitting the associated models, without considering the uncertainty for the measured quantities as derived in the previous section.

\subsection{Data reduction}

All data are non-dimensionalized according to the maneuvering convention. An example of the results processing is given in Fig. 7 including derived quantities and associated uncertainties. A complete record is included in the data archive [10].

$C_{X}=\frac{X}{\frac{1}{2} \rho V^{2} L T}$,
$C_{\mathrm{Y}}=\frac{Y}{\frac{1}{2} \rho V^{2} L T}$

$C_{\mathrm{N}}=\frac{N}{\frac{1}{2} \rho V^{2} L^{2} T}$.

The non-dimensionalization of all results is based on (constant) hull geometry. This choice introduces and inconsistency when interpreting the behavior for coefficients such as the effective draft, where the varying planform area of the appendage is not included. An alternate data reduction approach would consider the behavior for each appendage relative to the bare hull value. The difference could then be scaled by the appropriate planform area. As will be seen in the discussion of results, the fidelity of the measurement system is already limiting for the absolute quantities presented.
Table 1 Estimates for the precision limit (B-P3-L1-H3 (16), Fn $=0.21$, leeway angle $\beta=9^{\circ}$ )

\begin{tabular}{|c|c|c|c|c|c|c|}
\hline \multirow[t]{2}{*}{ Set } & \multicolumn{2}{|c|}{ Resistance } & \multicolumn{2}{|c|}{ Sideforce } & \multicolumn{2}{|c|}{ Yaw moment } \\
\hline & $\mu(\mathrm{N})$ & $U_{95}(\%)$ & $\mu(\mathrm{N})$ & $U_{95}(\%)$ & $\mu(\mathrm{Nm})$ & $U_{95}(\%)$ \\
\hline $\begin{array}{l}\text { Complete set } \\
\text { (Fig. 6) }\end{array}$ & 7.03 & 2.5 & 9.26 & 3.9 & 10.9 & 5.4 \\
\hline $\begin{array}{l}\text { First realization } \\
(15 / 12 / 2017)\end{array}$ & 6.97 & 2.1 & 9.17 & 4.0 & 10.7 & 2.8 \\
\hline $\begin{array}{l}\text { Second realization } \\
(7 / 1 / 2018)\end{array}$ & 7.08 & 1.9 & 9.36 & 2.9 & 11.2 & 2.0 \\
\hline $\begin{array}{l}\text { Alignment frame } \\
\text { (Fig. 6a) }\end{array}$ & 7.02 & 1.9 & 9.26 & 2.9 & 10.9 & 4.8 \\
\hline
\end{tabular}

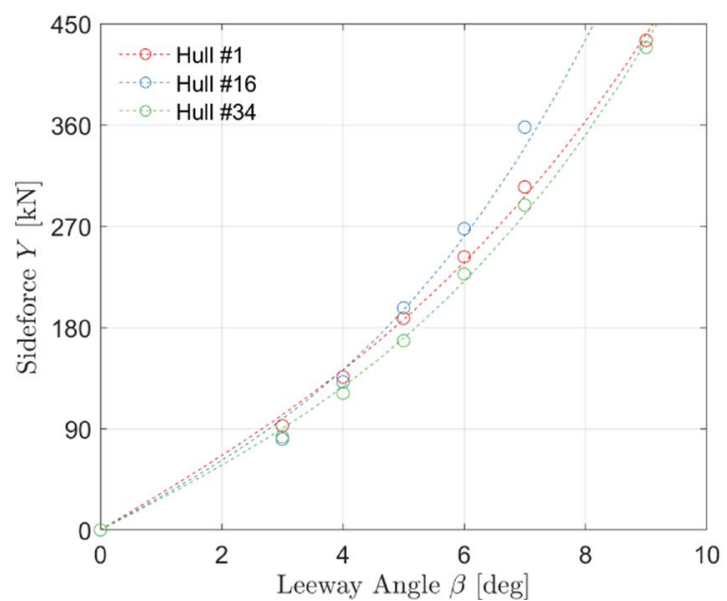

Fig. 8 Bare-hull sailing performance for Hulls \#1, \#16, and \#34. (left: sideforce, right: yaw moment) Fn $=0.21, \phi=0^{\circ}$. Simulation result 


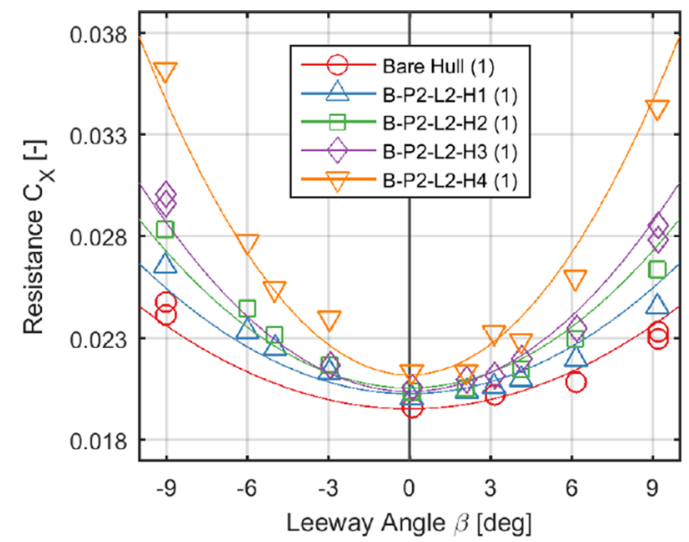

a Measured resistance data and fitted maneuvering model for variations in bilge keel height (Hull \#1).

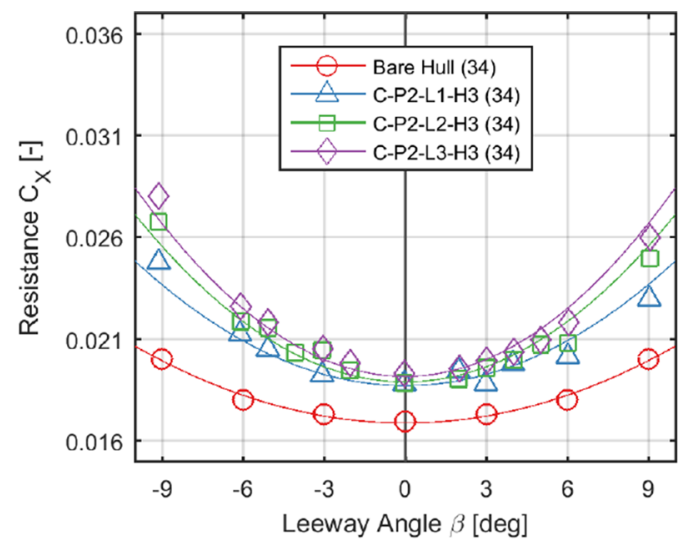

b Measured resistance data and fitted maneuvering model for variations in centerline keel length (Hull \#34).

Fig. 9 Vessel resistance for systematic variations in centerline keel length (left) and bilge keel height (right)

\subsubsection{Maneuvering coefficients}

The resistance, sideforce, and yaw moment are decomposed according to the maneuvering convention. A third-order model is assumed for the sideforce and yaw moment [5].

$C_{X}=X_{\beta \beta} \beta^{2}+C_{X O}$

$C_{\mathrm{Y}}=Y_{\text {lin }} \beta+Y_{\text {non-lin }} \beta^{3}$,

$C_{\mathrm{Y}}=Y_{\beta} \beta+Y_{\beta \beta \beta} \beta^{3}$,

$C_{\mathrm{N}}=N_{\text {lin }} \beta+N_{\text {non-lin }} \beta^{3}$,

$C_{\mathrm{N}}=N_{\beta} \beta+N_{\beta \beta \beta} \beta^{3}$.

\subsubsection{Sailing efficiency}

The resistance increase due to sideforce production is reported as an effective draft $T_{e}$, representing the efficiency for the sideforce production by the hull [25]. The effective draft of a sailing ship is the span of a foil with equivalent behavior for the induced resistance, as determined from the slope of $C_{X}$ vs. $C_{\mathrm{Y}}^{2}$ [15].

$$
\begin{aligned}
& X=\frac{1}{\pi \rho V^{2} T_{\mathrm{e}}^{2}} Y^{2}+R_{\mathrm{T}}, \\
& C_{X}=\frac{1}{\pi A R_{\mathrm{eff}}} C_{\mathrm{Y}}^{2}+C_{X O},
\end{aligned}
$$

and

$T_{\mathrm{e}}=\sqrt{\frac{T L}{2} A R_{\mathrm{eff}}}$.

The effective draft is presented in dimensionless form as $T_{\mathrm{e}} / T$.

\section{Discussion of sailing performance}

In the following discussion of the effective draft and CLR for systematic variations for bilge keel height (span), keel position, and keel length, the presentation of results includes

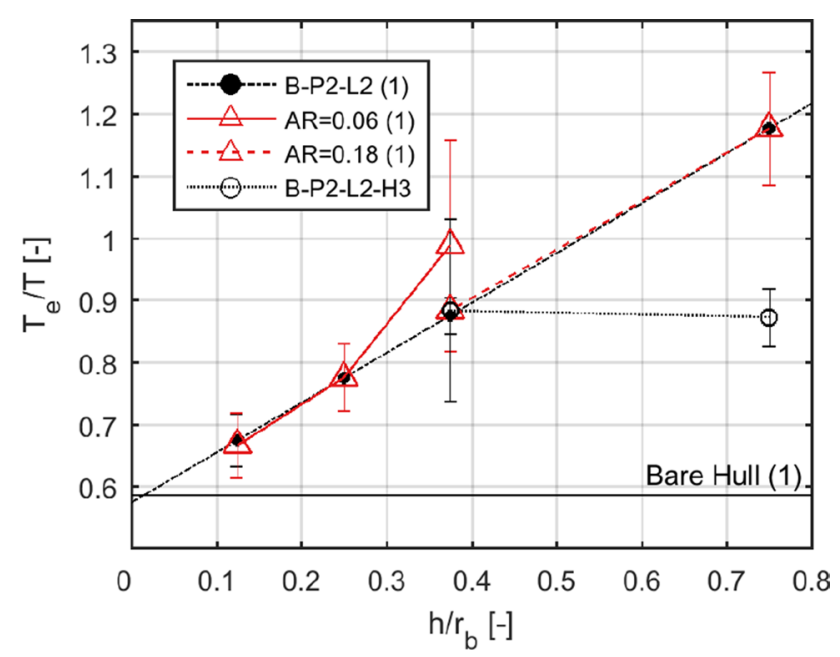

Fig. 10 Effective draft for variations in bilge keel height, aspect ratio, and $\frac{h}{R_{\mathrm{b}}}$ 

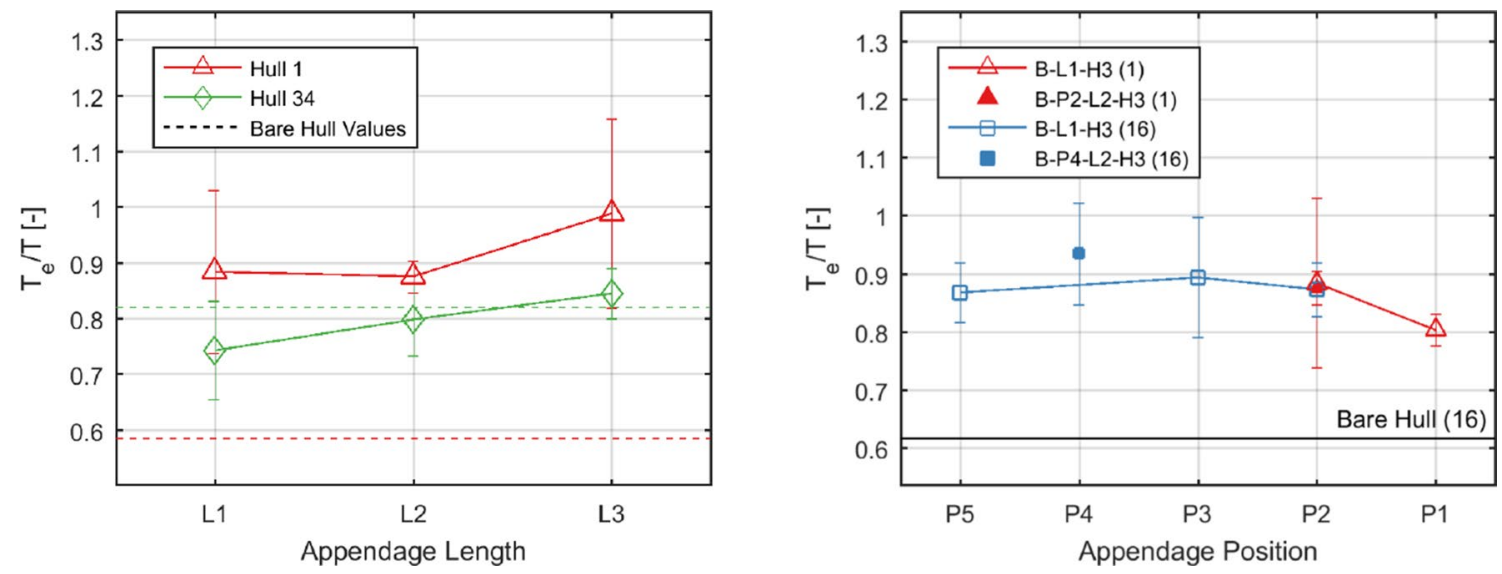

Fig. 11 Behavior for systematic variations in keel length (left) and position (right)

absolute and relative values. The combination of uncertainties in measured quantities and the added uncertainty associated with fitting results in large uncertainties. For relative quantities in particular, this must temper any conclusions made about trends in the data. Only linear coefficients for sideforce and yaw moment are considered in relative terms (Eq. 11). As a general statement, the substantial uncertainties as indicated in figures must temper any conclusions made (Fig. 8).

$\Delta Y_{\beta}=Y_{\beta}-\left.Y_{\beta}\right|_{\text {Bare }}$

$\Delta N_{\beta}=N_{\beta}-\left.N_{\beta}\right|_{\text {Bare }}$

Bilge keel variants were tested on two hulls-Hull \#1 and Hull \#16-where Hull \#16 is a much fuller ship and has a $50 \%$ smaller bilge radius. Bare-hull sailing performance for Hull \#16, with lengthened parallel midbody and smaller bilge radius, is improved compared with Hull \#1 for all metrics of sailing performance (see Table 3 ). In particular, the non-linear component of both sideforce and yaw moment are increased (negatively in the case of the moment). Hull \#34 shows improved performance for the yaw coefficients in particular, as well as the effective draft. The uncertainties for this hull were somewhat larger due to a manufacturing error. The bare hull value is indicated in the figures throughout the subsequent discussion of results.

As expected, the introduction of bilge keels increases the resistance at $0^{\circ}$ leeway compared with the bare-hull value (Fig. 9a). For bilge keels of varying height, the increase is in proportion with the increased surface area. In the results for the resistance, an asymmetry for the positive and negative leeway angles is evident. It was not possible to discern an alignment fault based on the resistance measurements alone.

The bias indicated by the asymmetry for resistance values at leeway, observed in Fig. 9, is much larger than the alignment bias (see Fig. 6). This effect is persistent in the remainder of the data set for Hull \#34 (Fig. 9b), and less so for hulls \#1 (Fig. 9a and \#16, suggesting that the fault has two sources. On the one hand, there is apparently a misalignment in the measurement frame. Also, Hull \#34 was constructed with a geometric fault (the milling machine coordinate system was skewed by approximately $3 \mathrm{~mm}$ over $2.7 \mathrm{~m}$ model length). The resistance, and the induced resistance (resistance arising due to sideforce production in particular), presented the greatest measurement challenge for the experimental system.

Four sets of appendages are plotted in Fig. 10, where the $x$-axis has been scaled with the bilge radius. The set for pure variations in appendage height is displayed as filled black circles. In Fig. 10 for the effective draft, a linear fit is added to underscore the nearly perfect correlation between appendage height and effective draft. Two sets for constant bilge

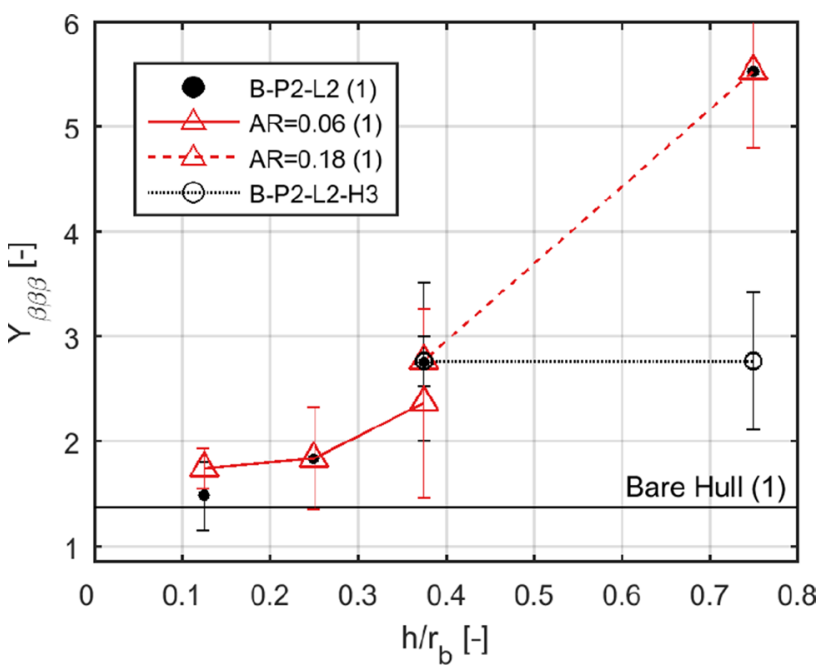

Fig. 12 Non-linear sideforce coefficient variations in bilge keel height 
keel aspect ratio are displayed as red triangles. Finally, the appendage geometry B-P2-L2-H3, which was tested on both hulls, is plotted as open black circles. Figure 11 contains results for variation in appendage position, tested on Hull \#1 and Hull \#16.

Two appendage sub-sets with constant aspect ratio are defined to test whether this class of appendages behaves as a normal lifting surface, for which the effective draft is expected to correlate with the appendage aspect ratio. Instead, the effective draft is nearly perfectly correlated with the results for variation in appendage height, a point that is underscored with a linear fit to the set B-P2-L2 (1) in
Fig. 10. The data reduction adopted for this presentation of results does not consider the varying planform area of the appendage. For this set, a scaling by the appendage planform area is equivalent to scaling by appendage height, meaning that this result is a constant response for effective draft. A similar scaling applied to the results for constant aspect ratio was attempted, but further analysis is needed.

A final set was defined for a variation in the ratio keel height to bilge radius, $\frac{h}{R_{\mathrm{b}}}$. The bilge keel B-P2-L2-H3 was tested on both Hull \#1 and Hull \#16, for which the bilge radius decreases by a factor of two (see Fig. 3). For both the quantities presented, and for the other maneuvering
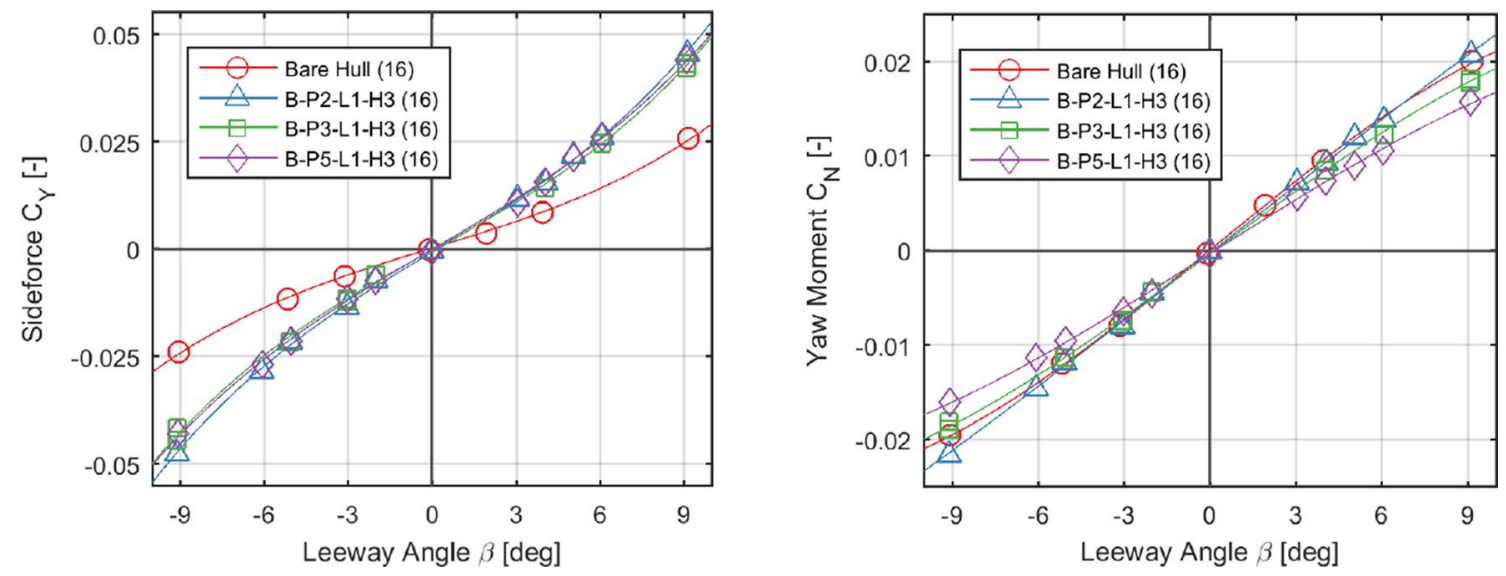

Fig. 13 Measured sideforce data and fitted maneuvering model for variations in bilge keel position

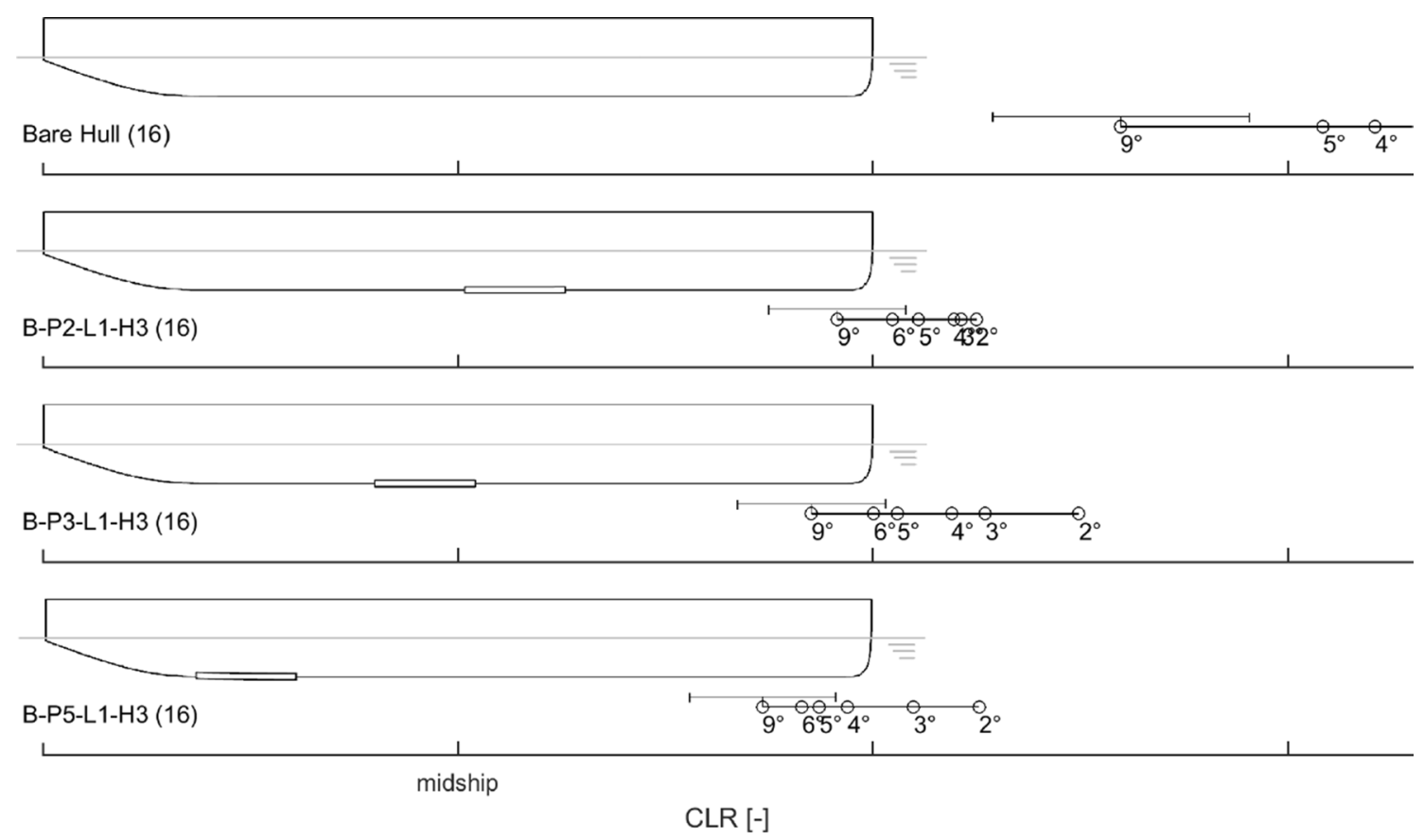

Fig. 14 The center of lateral resistance for variations in appendage position. The associated uncertainty for $9^{\circ}$ leeway is indicated for reference 
coefficients, the sailing performance is apparently independent of this ratio. It should be noted that Hull \#16 bare hull value for $Y_{\beta \beta \beta}$ is 1.67 , and $T_{\mathrm{e}} / T=0.62$ (see Table 3 ).

The effective draft is presented in Fig. 11, including the bare-hull value for reference. For both bilge keels and centerline keels, the trend is well evident: the coefficient appears to vary in proportion with the appendage length, a constant response when scaled by the appendage planform area. Whereas the sailing efficiency for a hull fitted with bilge keels increases by at least $50 \%$ over the bare hull value, and this value appears to increase for the longest bilge keel (L3), the centerline keel does not appear to improve the sailing efficiency of the hull. It must be noted that the uncertainty for the effective draft for results of Hull \#34 was such that these results were omitted from the journal publication. The effective draft as a function of bilge keel position does not appear to vary, especially in

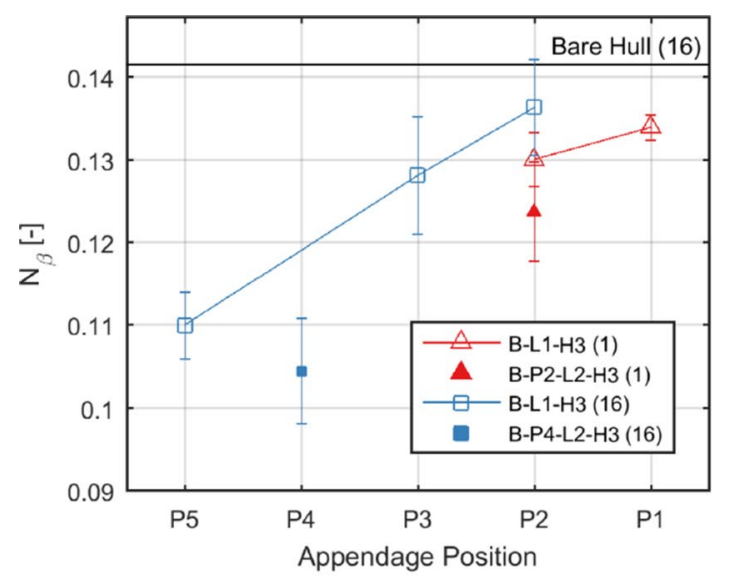

a Linear yaw moment $N_{\beta}$

Fig. 15 Yaw coefficients for variations in bilge keel position

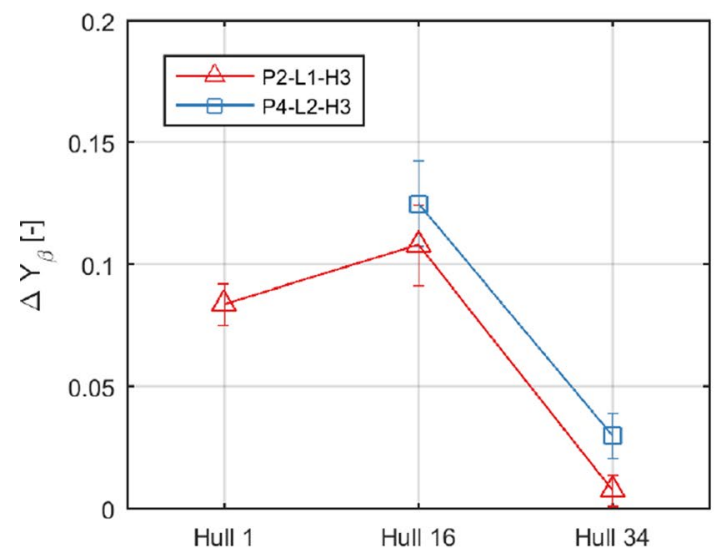

light of the uncertainties present. The results for Hull \#1 suggest that the forward keel positions are detrimental to the sailing efficiency.

As outlined under experimental design, the first objective for the set of varying appendage heights is to ascertain that the bilge keel behaves as expected within the model-scale boundary layer. It is understood that the bilge keel should act to promote flow separation along the leading bilge, contributing to the non-linear sideforce term. In Fig. 12 for the non-linear sideforce coefficient, the response in the neighborhood of the nominal bilge keel height for the experiment (H3) - corresponding to $h / r_{\mathrm{b}}=0.375$-is nearly linear with keel height, a result also reported by [11], whereas the behaviour at small values of $h / r_{\mathrm{b}}$ appears to approach the bare hull value asymptotically. Bilge keel H1 $\left(h / r_{\mathrm{b}}=0.11\right)$ is a geometric scaling for a representative full-scale bilge keel ( $h=0.5 \mathrm{~m}$ at full scale). This bilge keel height does

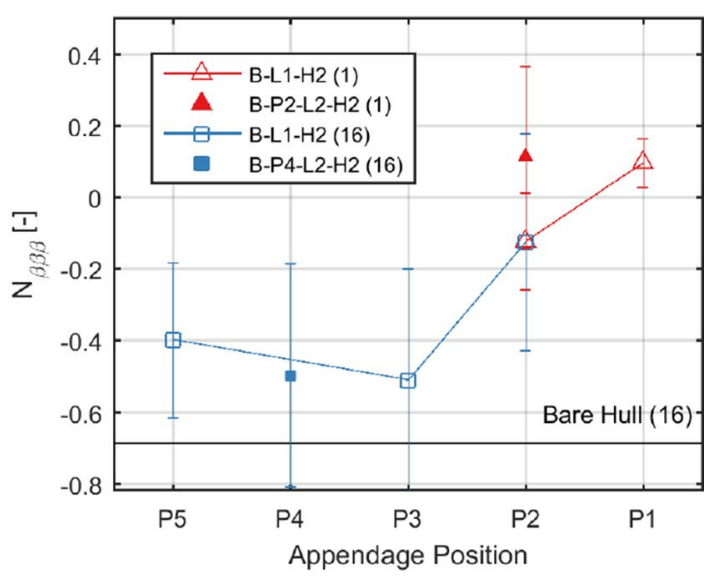

b Non-linear yaw moment $N_{\beta \beta \beta}$

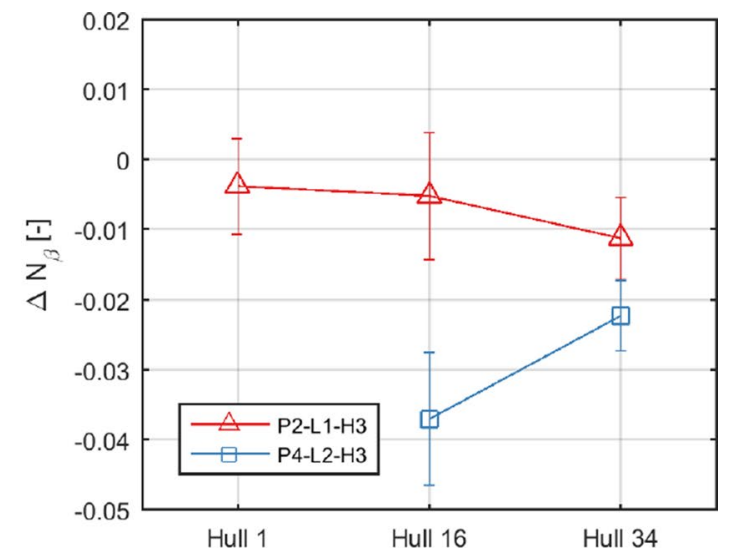

Fig. 16 Relative response for linear sideforce and yaw moment coefficient for identical bilge keel and centerline keel variants 
Table 2 Details for calculation of experimental uncertainty for resistance for keel variant B-P3-L1-H2 (16) at $\mathrm{Fn}=0.21$ and leeway angle $\beta=9^{\circ}$. The uncertainty $u^{\prime}$ is given as a percentage of the measured value. The geometric error is the dominant term

\begin{tabular}{lllll}
\hline$\Theta$ & $\frac{\partial X}{\partial \Theta}$ & $\delta_{\Theta}$ & $u_{\Theta}(\mathrm{N})$ & $u_{\Theta}^{\prime}(\%)$ \\
\hline$X$ & 0.0094 & $0.0423 \mathrm{~N}$ & 0.084 & 1.2 \\
$\beta$ & 0.0011 & $0.0029 \mathrm{rad}$ & $3.19 \mathrm{E}-6$ & 0.0 \\
$T$ & 0.0024 & $0.002 \mathrm{~m}$ & 0.185 & 2.6 \\
$P$ & & & 0.135 & 1.9 \\
\hline
\end{tabular}

Table 3 Sailing performance for un-appended hulls

\begin{tabular}{llllll}
\hline & $T_{\mathrm{e}} / T(-)$ & $Y_{\beta}(-)$ & $Y_{\beta \beta \beta}(-)$ & $N_{\beta}(-)$ & $N_{\beta \beta \beta}(-)$ \\
\hline Hull \#1-Bare & 0.59 & 0.09 & 1.37 & 0.13 & -0.25 \\
Hull \#16-Bare & 0.62 & 0.11 & 1.67 & 0.14 & -0.68 \\
Hull \#34-Bare & 0.82 & 0.088 & 2.61 & 0.12 & 0.03 \\
\hline
\end{tabular}

not excite the expected response for the non-linear sideforce term.

The measured data for sideforce and yaw moment are presented in Fig. 13, including fitted maneuvering models. The yawing moment is most influenced by variations in appendage position, whereas the sideforce is nearly insensitive. It is interesting to note the response for appendage P2-L1-H3 (the most forward position shown) in yaw, for which the non-linear behavior is reduced compared with the bare hull.

The corresponding vessel CLR is given in Fig. 14 for the same bilge keel variations. Approximately similar behavior is observed for keels located at P3 and P5, where the CLR values for the bilge keel P5-L1-H3 are shifted aftward. The result for the CLR of the bilge keel at P2 is exceptional in that the grouping for varying leeway angles is much more compact than the other cases. A small variation for CLR with leeway angle indicates that the distribution of lateral force along the hull is steady as the leeway angle changes.
Table 4 Complete array of testing matrix

\begin{tabular}{|c|c|c|c|c|c|}
\hline Keel set & Identifier & Position (mm) & Length (mm) & Height (mm) & Hull \\
\hline \multirow[t]{3}{*}{$\mathrm{f}$ (length) —Bilge keel } & B-P2-L1-H3 (1) & 1735 & 333 & 30 & 1 \\
\hline & B-P2-L2-H3 (1) & 1735 & 667 & 30 & 1 \\
\hline & B-P2-L3-H3 (1) & 1735 & 1000 & 30 & 1 \\
\hline \multirow[t]{3}{*}{ f(length) - Centerline keel } & C-P2-L1-H3 (34) & 1735 & 333 & 30 & 34 \\
\hline & C-P2-L2-H3 (34) & 1735 & 667 & 30 & 34 \\
\hline & C-P2-L3-H3 (34) & 1735 & 1000 & 30 & 34 \\
\hline \multirow[t]{9}{*}{ f(position) } & B-P1-L1-H3 (1) & 2035 & 333 & 30 & 1 \\
\hline & B-P2-L1-H3 (1) & 1735 & 333 & 30 & 1 \\
\hline & B-P2-L1-H3 (16) & 1735 & 333 & 30 & 16 \\
\hline & C-P2-L1-H3 (34) & 1735 & 333 & 30 & 34 \\
\hline & B-P3-L1-H3 (16) & 1435 & 333 & 30 & 16 \\
\hline & B-P5-L1-H3 (16) & 835 & 333 & 30 & 16 \\
\hline & B-P2-L2-H3 (1) & 1735 & 667 & 30 & 1 \\
\hline & B-P4-L2-H3 (16) & 1135 & 667 & 30 & 16 \\
\hline & C-P4-L2-H3 (34) & 1135 & 667 & 30 & 34 \\
\hline \multirow[t]{4}{*}{ f(height) } & B-P2-L2-H1 (1) & 1735 & 667 & 10 & 1 \\
\hline & B-P2-L2-H2 (1) & 1735 & 667 & 20 & 1 \\
\hline & B-P2-L2-H3 (1) & 1735 & 667 & 30 & 1 \\
\hline & B-P2-L2-H4 (1) & 1735 & 667 & 60 & 1 \\
\hline \multirow[t]{3}{*}{$\mathrm{f}(\mathrm{AR}=0.06)$} & B-P2-L1-H1 (1) & 1735 & 333 & 10 & 1 \\
\hline & B-P2-L2-H2 (1) & 1735 & 667 & 20 & 1 \\
\hline & B-P2-L3-H3 (1) & 1735 & 1000 & 30 & 1 \\
\hline \multirow[t]{2}{*}{$\mathrm{f}(\mathrm{AR}=0.18)$} & B-P2-L1-H3 (1) & 1735 & 333 & 30 & 1 \\
\hline & B-P2-L2-H4 (1) & 1735 & 667 & 60 & 1 \\
\hline \multirow[t]{2}{*}{$f\left(h / h_{\mathrm{b}}\right)$} & B-P2-L1-H3 (1) & 1735 & 333 & 30 & 1 \\
\hline & B-P2-L1-H3 (16) & 1735 & 333 & 30 & 16 \\
\hline \multirow[t]{5}{*}{ f(CL keel) } & C-P2-L1-H3 (34) & 1735 & 333 & 30 & 34 \\
\hline & B-P2-L1-H3 (1) & 1735 & 333 & 30 & 1 \\
\hline & B-P2-L1-H3 (16) & 1735 & 333 & 30 & 16 \\
\hline & C-P4-L2-H3 (34) & 1135 & 667 & 30 & 34 \\
\hline & B-P4-L2-H3 (16) & 1135 & 667 & 30 & 16 \\
\hline
\end{tabular}




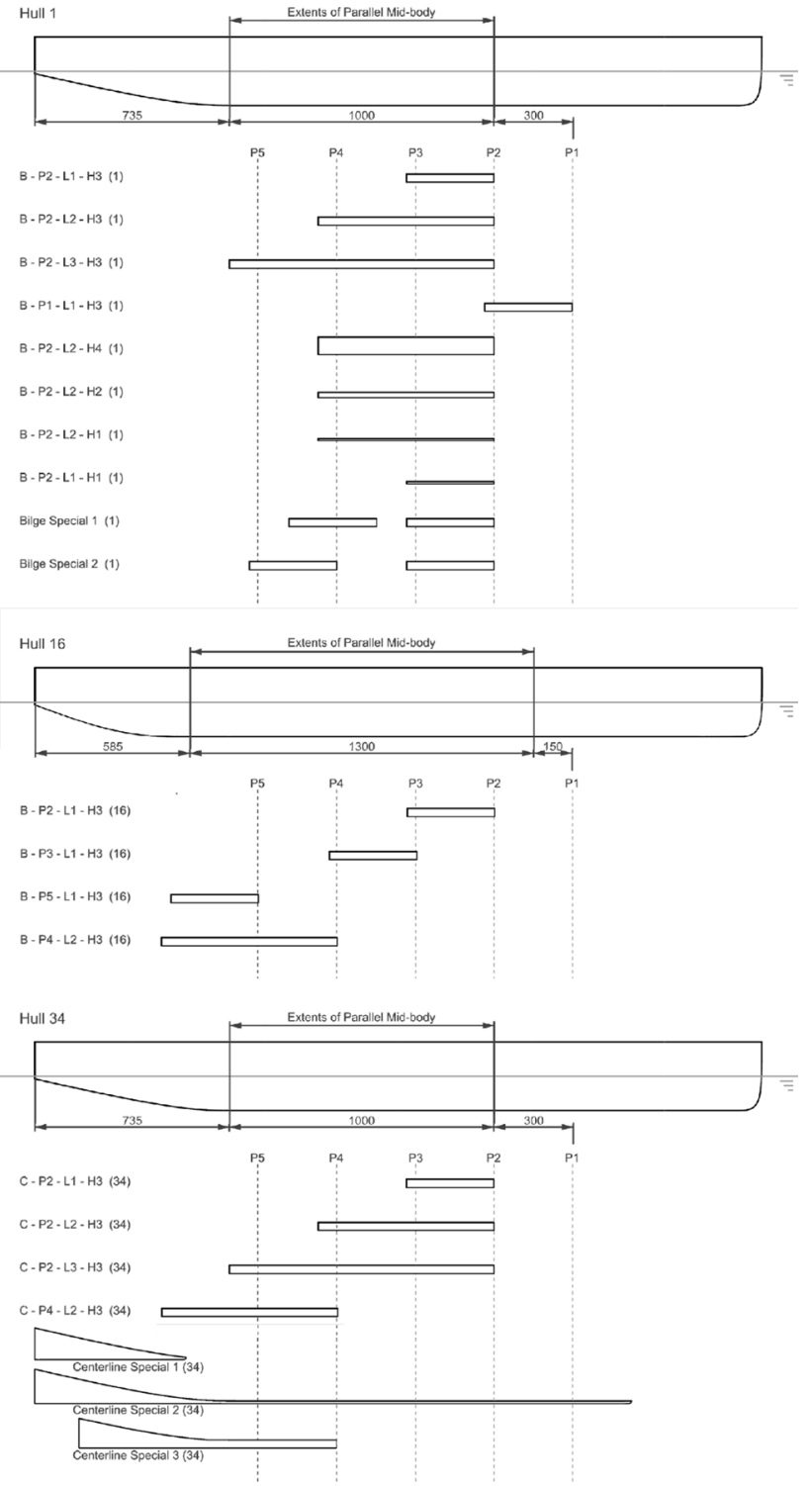

Fig. 17 Complete array of tested keels

Following the work of [19], the separation location for the bilge vortex associated with the non-linear sideforce term will shift along the leading bilge according to the leeway angle. Variation in CLR with leeway angle is in part due to this effect. A second phenomenon at play is the attenuation of the Munk yawing moment by separation effects along the aft body. Both effects are associated with the separation of bilge vortices, and are confined to larger leeway angles for the bare hull case.

The linear and non-linear maneuvering coefficients for the yaw moment are plotted in Fig. 15a, b, together with the bare hull value for Hull \#16 as reference. Besides the results for the appendage set presented above, data for appendages tested on Hull \#1 are included, as well as two cases for appendage length $\mathrm{L} 2$ with solid markers. The trend observed in the raw data is clearly evident here: as the bilge keel is moved aft, the linear yaw moment is reduced. The results for the non-linear coefficient are subject to the same qualifications as for the non-linear sideforce above, besides which, the non-linearity for the yaw moment is a rather small effect. Nevertheless, the response is markedly different for forward keel positions P1 and P2 (for reference, Hull \#1 bare hull value for $N_{\beta \beta \beta}$ is -0.25 ).

Finally, results for identical bilge keel and centerline keel variants are presented together in Fig. 16. One can observe the systemic increase reported also by [48] for sideforce $Y_{\beta}$; however, in contrast, the yawing moment $\left(N_{\beta}\right)$ is reduced. Following the discussion under Fig. 15a, a reduction in the yawing moment is expected for small drifting angles. This behaviour is driven by a release or attenuation of the Munk moment underpressure along the aft-side, the keel at after position P4 seeing the largest decrease (Figs. 17, 18).

\section{Conclusion}

The working principle for bilge keels is the promotion of flow separation. For sailing ships, placement of bilge keels raises the possibility of specifying the separation location and of inducing flow separation at leeway angles for which separation would not yet occur for the un-appended hull. Results for small keel heights affirm the dimensioning adopted for the test, where a simple geometric scaling would not be appropriate. The behaviour observed in the neighborhood of the nominal height, set such that the keel extends unambiguously through the entire height of the model-scale boundary layer, conforms to results reported by [11]. There is a linear relationship between bilge keel height and the coefficients for sideforce and sailing efficiency (indicating a constant behaviour if the appendage planform area is considered). In contrast with the bilge heel height, bilge keel position influences both the linear and non-linear coefficients for the yawing moment, rather than the sideforce strength. One exceptional result to highlight is the keel at position P2 (approximately midship), for which the variation in CLR with leeway angle is markedly reduced. The yaw response is nearly linear for appendages at positions P1 and P2 (linear behaviour for both sideforce and yaw would return a CLR with no variation for leeway angle). Response for bilge keel length is restricted to sideforce, whereas yaw moment is unaffected. As for the bilge keel height, the linear response for the linear coefficient implies a constant response if the appendage planform area is taken into account (Tables 4,5).

Bilge keels and centerline keels are categorized as boundary-layer appendages, where the working principle is the promotion of flow separation in the vessel boundary layer. The appendage typology is shown to mitigate the strong 


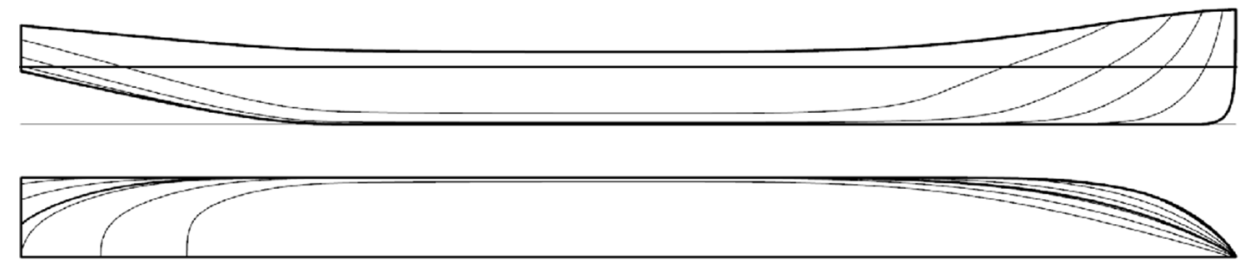

Hull \#1: Parent hull for First Series
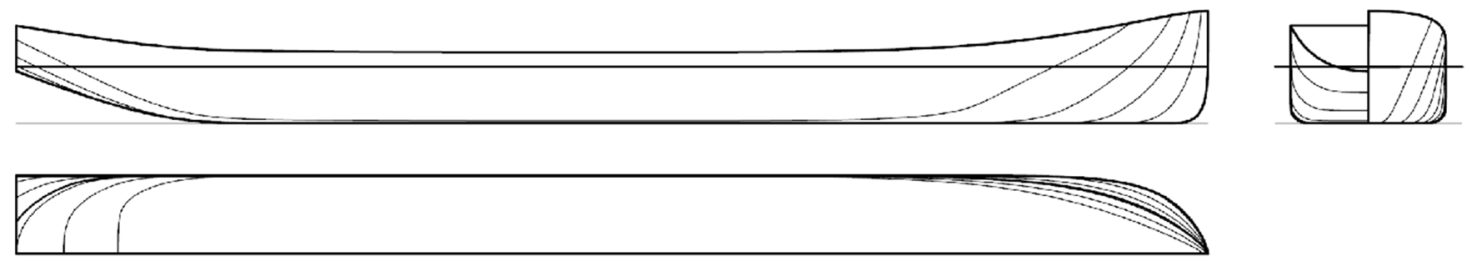

Hull \#16: Full-bodied with sharp bilges
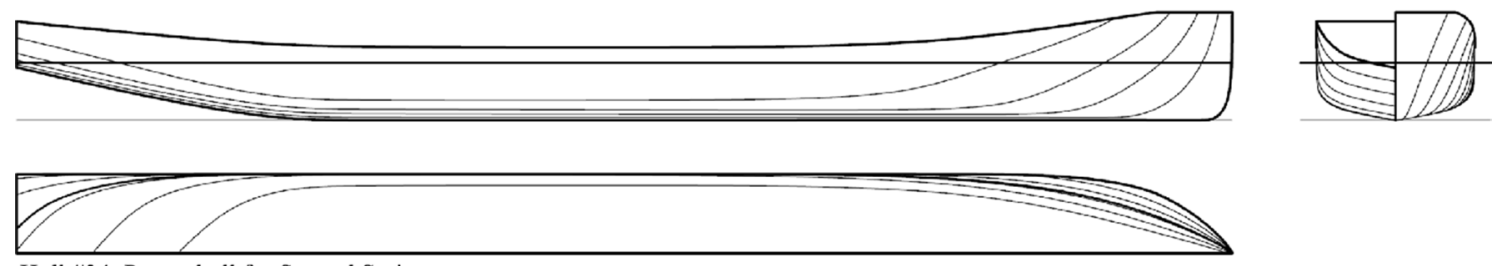

Hull \#34: Parent hull for Second Series

Fig. 18 Lines plan for Hulls \#1, \#16, and \#34

Table 5 Main particulars for Hulls 1, 16, and 34

\begin{tabular}{lllll}
\hline & Hull \#1 & Hull \#1 & $\begin{array}{l}\text { Hull \#16 } \\
\text { Full scale }\end{array}$ & $\begin{array}{l}\text { Hodl \#34 } \\
\text { Model: } \alpha=50\end{array}$ \\
& Model & Model \\
\hline LOA (m) & 138 & 2.76 & 2.76 & 2.76 \\
Beam (m) & 18 & 0.36 & 0.36 & 0.36 \\
Draft (m) & 6.5 & 0.13 & 0.13 & 0.13 \\
Displacement & $11,896 \mathrm{t}$ & $92.8 \mathrm{~kg}$ & $106.6 \mathrm{~kg}$ & $84.8 \mathrm{~kg}$ \\
$C_{\mathrm{B}}$ & 0.719 & 0.719 & 0.826 & 0.641 \\
$C_{\mathrm{P}}$ & 0.764 & 0.764 & 0.840 & 0.764 \\
$C_{\mathrm{M}}$ & 0.942 & 0.942 & 0.984 & 0.838 \\
$L C B(\%)$ & 50.13 & 50.13 & 49.7 & 50.2 \\
Whetted surface $\mathrm{m}^{2}$ & 3.293 & 1.312 & 1.453 & 1.269 \\
\hline
\end{tabular}

Munk yawing moment that is characteristic of wind-assisted commercial vessels, and to promote the non-linear sideforce component. Also, the sailing efficiency (resistance increase due to sideforce) of a hull fitted with bilge keels or centerline keels, presenting a sharp tip profile to the lateral flow, can be improved dramatically. An optimal arrangement of bilge keels will result in a pattern of separation that is beneficial to the course-keeping of the vessel and its sailing efficiency. Experimental results from towing tank experiments for the sailing performance of bilge keels, centerline keels, and other special typologies have been presented. The bilge keel, already commonplace in the naval architecture of ships, finds new purpose as an effective appendage for wind-assisted commercial ships.

Electronic supplementary material The online version of this article (https://doi.org/10.1007/s00773-020-00777-8) contains supplementary material, which is available to authorized users.

Acknowledgements The author gratefully acknowledges the contribution from Dykstra Naval Architects when planning this experiment. This research was funded by the Bijlboeg Fonds and the Samenwerkende Maritieme Fondsen of The Netherlands.

Open Access This article is licensed under a Creative Commons Attribution 4.0 International License, which permits use, sharing, adaptation, distribution and reproduction in any medium or format, as long as you give appropriate credit to the original author(s) and the source, provide a link to the Creative Commons licence, and indicate if changes were made. The images or other third party material in this article are included in the article's Creative Commons licence, unless indicated otherwise in a credit line to the material. If material is not included in the article's Creative Commons licence and your intended use is not permitted by statutory regulation or exceeds the permitted use, you will need to obtain permission directly from the copyright holder. To view a copy of this licence, visit http://creativecommons.org/licenses/by/4.0/. 


\section{References}

1. Abdel-Maksoud M et al (2016) Experimental and numerical investigations on flow characteristics of the $\mathrm{KVLCC} 2$ at $30^{\circ}$ drift angle. In: SNAME transactions, $\mathrm{p} 26$

2. Beukelman W (1993) Lift and drag for a low aspect-ratio surface piersing wing-model in deep and shallow water. Delft University of Technology, technical report

3. Beukelman W (1998) Manoeuvring coefficients for a wing-model in deep and shallow water. Int Shipbuild Prog 45:441

4. Beukelman W (1989) Cross Fow drag on a segmented model. Technical report, TU Delft

5. Beukelman W, Journée JMJ (2001) Hydrodynamic transverse loads on ships in deep and shallow water. In: HADMAR'2001, 22nd international conference hydrodynamics aerodynamics

6. Braathen A (1987) Application of a vortex tracking method to the prediction of roll damping. In: Norwegean Institute Technology University TRONDHEIM

7. Carrica PM et al (2016) Direct simulation and experimental study of zigzag maneuver of KCS in shallow waterx. Ocean Eng 112:117-133

8. Claughton AR, Oliver JC (2003) Developments in hydrodynamic force models for velocity prediction programs. In: Conference on the modern Yacht. Royal Institute of Naval Architects

9. Coleman HW, Steele WG (1990) Experimentation and uncertainty analysis for engineers. Sterling 6(3):231-231. https://doi. org/10.1002/qre.4680060315

10. van Der Kolk NJ et al (2019) Case study: wind-assisted ship propulsion performance prediction, routing, and economic modelling. In: Proceedings of the international conference power \& propulsion alternatives for ships. The Royal Institution of Naval Architects, London

11. Faltinsen OM, Sortland B (1987) Slow drift eddy making damping of a ship. Appl Ocean Res 9(1):37-46

12. Froude $W$ (1865) On the practical limits of the rolling of a ship in a seaway. Trans Inst Nav Archit 6

13. Fujiwara T, Ueno M, Ikeda Y (2005) A new estimation method of wind forces and moments acting on ships on the basis of physical component models. J Jpn Soc Nav Archit Ocean Eng 2:243-255

14. Fujiwara et al T (2003) On aerodynamic characteristics of a hybrid-sail with square soft sail. In: Thirteenth international conference for offshore polar engineering

15. Gerritsma J, Keuning JA (1992) Sailing yacht performance in calm water and in waves. In: HISWA symposium on yacht design and yacht construction. Amsterdam, pp. 233-245

16. Gerritsma J, Keuning JA, Versluis A (1993) Sailing yacht performance in calm water and in waves. TU Delft Report No. 938-P. January 1993

17. Hoerner SF (1965) Fluid dynamic lift-practical information on aerodynamic drag and hydrodynamic resistance. STIA, Bakersfield

18. Hoerner SF (1985) Fluid dynamic lift—practical information on aerodynamic and hydrodynamic lift. Mrs. Liselotte A. Hoerner, Vancouver

19. Hooft JP (1994) Cross flow drag on a manoeuvring ship. Ocean Eng. https://doi.org/10.1016/0029-8018(94)90004-3

20. Hooft JP, Quadvlieg F (1996) Non-linear hydrodynamic hull forces derived from segmented model tests. In: In Marine simulation and ship manoeuvrability, pp 399-409

21. ITTC (2008) Force and moment uncertainty analysis for planar motion test. Technical report

22. Ikeda N, Himeno Y, Tanaka (1976) OnRoll Damping Force of Ship: Effects ofFriction of Hull and Normal Force of BilgeKeels. J Kansai Soc Nav Archit 161:41-49
23. Jacobs WR (1964) Estimation of stability derivatives and indices of various ship forms, and comparison with experimental results. J Ship Res 12:135-163

24. Jones RT (1946) Properties of low-aspect ratio pointed wings at speeds below and above the speed of sound. Technical report 835 . NACA

25. Keuning JA, Binkhorst BJ (1997) Appendage resistance of a sailing yacht hull. In: Chesapeak sail yacht symposium

26. Keuning JA, Vermeulen KJ (2002) On the balance of large sailing yachts. In: HISWA symposium on yacht design and yacht construction. Amsterdam

27. Kijima K, Nakiri Y (1990) Prediction method of ship manoeuvrability in deep and shallow waters

28. van der Kolk NJ et al. (2019) Dataset: bilge keels for course stability and 640 sailing efficiency of wind-assisted ships.en. https://doi. org/10.4121/UUID:A9314E24-5D4C-4C46-9F99-391EE7CDC1 EB

29. Kramer JA, Steen S, Savio L (2016) Experimental study of the effect of drift angle on a ship-like foil with varying aspect ratio and bottom edge shape. Ocean Eng 121:530-545

30. Longo J, Stern F (2002) Effects of drift angle on model ship flow. Exp Fluids 32(5):558-569

31. Mander S (2017) Slow steaming and a new dawn for wind propulsion: a multilevel analysis of two low carbon shipping transitions. Maritime Policy 75:210-216

32. Minami $Y$ et al. (2003) Investigation into underwater fin arrangement effect on steady sailing characteristics of a sail assisted ship. In: International offshore and polar engineering conference-proceedings, pp 318-325

33. Munk MM (1924) Remarks on the pressure distribution over the surface of an ellipsoid moving translationally through a perfect fluid. Technical report, NACA

34. Newman JN (1977) Marine hydrodynamics. MIT Press, Boston

35. Nomoto K, Tatano H (1979) "Balance of Helm of sailing Yachts". In: HISWA Sailing Yacht Symposium, pp 65-89.

36. Ouchi K et al (2013) 'Wind Challenger' the next generation hybrid sailing vessel. University of Tokyo, technical report

37. Prandtl L (1918) Tragflügeltheorie. In: Königliche Gesellschaft der Wissenschaften zu Göttingen

38. Sarpkaya T, O'Keefe JL (1996) Oscillating flow about two and three-dimensional bilge keels. J. Offshore Mech Arct Eng 118:1-6

39. Satchwell CJ (1985) Windship technology. In: Proceedings of the International Symposium on Windship Technology (WINDTECH '85), Vol 19, Amsterdam, Elsevier

40. Skogman A (1985) The practical meaning of lateral balance for a sail-assisted research vessel. J Wind Eng Ind Aerodyn 20(1):201-226

41. Smith T et al (2013) Analysis techniques for evaluating the fuel savings associated with wind assistance. In: Low carbon shipping conference

42. Traut $\mathrm{M}$ et al (2018) $\mathrm{CO} 2$ abatement goals for international shipping. Clim Policy 18(8):1066-1075

43. Michael T et al (2014) Propulsive power contribution of a kite and a Flettner rotor on selected shipping routes. Appl Energy 113:362-372. https://doi.org/10.1016/j.apenergy.2013.07.026

44. Tsakonas S (1959) Effect of appendage and hull form on hydrodynamic coefficients of surface ships. Technical report, Hoboken, $\mathrm{NJ}$

45. van't Veer R, Schut X, Pelerin J (2015) Bilge keel loads and hull induced pressures: experimental results. Omae2015

46. Walsh C, Mander S, Larkin A (2017) Charting a low carbon future for shipping: a UK perspective. Maritime Policy 82:32-40

47. Xing T, Bhushan S, Stern F (2012) Vortical and turbulent structures for KVLCC2 at drift angle 0, 12, and 30 degrees. Ocean Eng $55: 23-43$ 
48. Yasukawa H, Hirata N, Yamazaki Y (2017) Effect of bilge keels on maneuverability of a fine ship. J Mar Sci Technol 23(2):302-318. https://doi.org/10.1007/s00773-017-0474-6

49. Yoshimura Y (2002) A prospect of sail-assisted fishing boats. Fish Sci 68(2):1815-1818
Publisher's Note Springer Nature remains neutral with regard to jurisdictional claims in published maps and institutional affiliations. 